\title{
2009s-17
}

\section{Nonlinearity and Temporal Dependence}

\author{
Xiaohong Chen, Lars P. Hansen, \\ Marine Carrasco
}

Série Scientifique
Scientific Series

\author{
Montréal \\ Mai 2009
}

(C) 2009 Xiaohong Chen, Lars P. Hansen and Marine Carrasco. Tous droits réservés. All rights reserved. Reproduction partielle permise avec citation du document source, incluant la notice (C)

Short sections may be quoted without explicit permission, iffull credit, including (C notice, is given to the source.
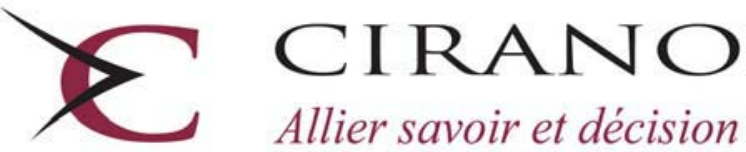

Allier savoir et décision

Centre interuniversitaire de recherche en analyse des organisations 


\section{CIRANO}

Le CIRANO est un organisme sans but lucratif constitué en vertu de la Loi des compagnies du Québec. Le financement de son infrastructure et de ses activités de recherche provient des cotisations de ses organisations-membres, d'une subvention d'infrastructure du Ministère du Développement économique et régional et de la Recherche, de même que des subventions et mandats obtenus par ses équipes de recherche.

CIRANO is a private non-profit organization incorporated under the Québec Companies Act. Its infrastructure and research activities are funded through fees paid by member organizations, an infrastructure grant from the Ministère du Développement économique et régional et de la Recherche, and grants and research mandates obtained by its research teams.

\section{Les partenaires du CIRANO}

Partenaire majeur

Ministère du Développement économique, de l'Innovation et de l'Exportation

\section{Partenaires corporatifs}

Banque de développement du Canada

Banque du Canada

Banque Laurentienne du Canada

Banque Nationale du Canada

Banque Royale du Canada

Banque Scotia

Bell Canada

BMO Groupe financier

Caisse de dépôt et placement du Québec

DMR

Fédération des caisses Desjardins du Québec

Gaz de France

Gaz Métro

Hydro-Québec

Industrie Canada

Investissements PSP

Ministère des Finances du Québec

Power Corporation du Canada

Raymond Chabot Grant Thornton

Rio Tinto Alcan

State Street Global Advisors

Transat A.T.

Ville de Montréal

\section{Partenaires universitaires}

École Polytechnique de Montréal

HEC Montréal

McGill University

Université Concordia

Université de Montréal

Université de Sherbrooke

Université du Québec

Université du Québec à Montréal

Université Laval

Le CIRANO collabore avec de nombreux centres et chaires de recherche universitaires dont on peut consulter la liste sur son site web.

Les cahiers de la série scientifique (CS) visent à rendre accessibles des résultats de recherche effectuée au CIRANO afin de susciter échanges et commentaires. Ces cahiers sont écrits dans le style des publications scientifiques. Les idées et les opinions émises sont sous l'unique responsabilité des auteurs et ne représentent pas nécessairement les positions du CIRANO ou de ses partenaires.

This paper presents research carried out at CIRANO and aims at encouraging discussion and comment. The observations and viewpoints expressed are the sole responsibility of the authors. They do not necessarily represent positions of CIRANO or its partners. 


\title{
Nonlinearity and Temporal Dependence*
}

\author{
Xiaohong Chen $^{\dagger}$, Lars P. Hansen ${ }^{*}$, Marine Carrasco $^{\S}$
}

\begin{abstract}
Résumé / Abstract
Les non-linéarités dans les coefficients de mouvement et de diffusion ont une incidence sur la dépendance temporelle dans le cas des modèles de diffusion scalaire. Nous examinons ce lien en recourant à deux notions de dépendance temporelle : mélange $\beta$ et mélange $\rho$. Nous démontrons que le mélange $\beta$ et le mélange $\rho$ avec dégradation exponentielle constituent des concepts fondamentalement équivalents en ce qui a trait aux diffusions scalaires. Pour ce qui est des diffusions stationnaires qui ne se classent pas dans le mélange $\rho$, nous démontrons qu'elles appartiennent quand même au mélange $\beta$, sauf que les taux de dégradation sont lents plutôt qu'exponentiels. Pour ce genre de processus, nous recourons à des transformations des états de Markov dont les variations sont finies, mais dont les densités spectrales sont infinies à la fréquence zéro. Certains états ont des densités spectrales qui divergent à la fréquence zéro de la même façon que dans le cas des processus stochastiques à mémoire longue. En terminant, nous indiquons la façon dont l'échantillonnage de Poisson qui est non linéaire et dépendant de l'état modifie la distribution inconditionnelle et la dépendance temporelle.
\end{abstract}

Mots clés : mélange, diffusion, forte dépendance, mémoire longue, échantillonnage de Poisson.

Nonlinearities in the drift and diffusion coefficients influence temporal dependence in scalar diffusion models. We study this link using two notions of temporal dependence: $\beta$-mixing and $\rho$-mixing. Weshow that $\beta$-mixing and $\rho$-mixing with exponential decay are essentially equivalent concepts for scalar diffusions. For stationary diffusions that fail to be $\rho$-mixing, we show that they are still $\beta$-mixing except that the decay rates are slower than exponential. For such processes we find transformations of the Markov states that have finite variances but infinite spectral densities at frequency zero. Some have spectral densities that diverge at frequency zero in a manner similar to that of stochastic processes with long memory. Finally we show how nonlinear, state-dependent, Poisson sampling alters the unconditional distribution as well as the temporal dependence.

Keywords: Mixing, Diffusion, Strong dependence, Long memory, Poisson sampling.

Codes JEL : C12, C13, C22, C50

\footnotetext{
${ }^{*}$ We thank a co-editor, an associate editor and two anonymous referees for valuable suggestions which greatly improved the paper. We also thank Rabi Bhattacharya, Richard Bradley, Javier Hidalgo, Jose Scheinkman and Noah Williams for helpful conversations, Noah Williams for research assistance, and participants of 2000 World Congress of the Econometric Society for comments. Chen and Hansen gratefully acknowledge support from the National Science Foundation.

${ }^{\dagger}$ Corresponding author: Department of Economics, Yale University, Box 208281, New Haven, CT 06520-8281, USA. Phone (203) 432-5852, Fax (203) 432-6167. Email: xiao-hong.chen@yale.edu.

${ }^{*}$ Departments of Economics and Statistics, University of Chicago.

${ }^{\S}$ Department of Economics, University of Montreal and CIRANO.
} 


\section{Introduction}

This paper studies how nonlinearity induces temporal dependence in continuous-time Markov models. Our interest in temporal dependence stems from a variety of empirical evidence.

Bond prices are known to be highly persistent and the conditional volatilities of financial time series are often temporally clustered, e.g., see Ding, Granger, and Engle (1993), Bollerslev and Mikkelsen (1996), and Andersen, Bollerslev, Diebold, and Labys (2001). Using linear time series methods, many researchers have documented the presence of long memory in empirical descriptions of data. Here we investigate when high degrees of temporal dependence are present in first-order and hence finite memory Markov models. In these models the dependence emerges because of the nonlinearities in the evolution equation for the Markov state.

Since hedging and pricing theory in financial economics often assume securities follow continuous-time nonlinear diffusions, we use these models as our way to capture nonlinearities in time series. For pedagogical and analytical convenience, we treat the case of scalar diffusions. That is, we study the solution to a stochastic differential equation:

$$
d x_{t}=\mu\left(x_{t}\right) d t+\sigma\left(x_{t}\right) d W_{t}
$$

where $\mu$ is the drift coefficient, $\sigma^{2}$ is the diffusion coefficient and $\left\{W_{t}\right\}$ is a one-dimensional standard Brownian motion. While scalar diffusion processes are special, they provide a very nice laboratory for our analysis. They allow for nonlinearities in the time series to be captured fully by two functions: the drift and the diffusion coefficients. By focusing on diffusion models, we are able to show clearly the mechanism whereby nonlinearities in a time series get transmitted into temporal dependence.

We classify the temporal dependence of scalar diffusions using two alternative notions of mixing: $\rho$-mixing and $\beta$-mixing. The $\rho$-mixing coefficients measure the temporal decay of maximally autocorrelated (nonlinear) functions of the Markov state. When $\rho-$ mixing coefficients decay to zero, the spectral density of any process with finite second moments formed by taking a nonlinear function of the state has a continuous (and hence finite) spectral density at all frequencies, including frequency zero. Thus $\rho$-mixing gives an operational way to classify the dependence of a nonlinear Markov process. The process is weakly dependent if the $\rho$-mixing coefficients decay to zero and strongly dependent if the $\rho$-mixing coefficients are identically one. In our study of strongly dependent processes, we also use $\beta$ - mixing, which for our purposes is a more refined concept. The $\beta-$ mixing coefficients for the strongly dependent processes have a decay rate that is slower than exponential and the implied spectral density functions for some of these processes diverge at frequency zero.

For both notions of mixing we are lead to use:

$$
\frac{\mu}{\sigma}-\frac{\sigma^{\prime}}{2}
$$

to measure the pull from the boundaries. When this pull measure is negative at the right boundary and positive at the left boundary, Hansen and Scheinkman (1995) have shown how the non-zero boundary pull restrictions imply $\rho$-mixing with exponential decay. We extend their result by showing that in the case of scalar diffusions, $\beta$ - mixing and $\rho$-mixing with exponential decay are almost equivalent concepts, and both are implied by the non-zero boundary pull restrictions.

When the pull measure is zero at one of the boundaries, the Markov process is strongly dependent: the $\rho$-mixing coefficients are identically one and the $\beta$ - mixing coefficients decay at a rate slower than exponential. We provide sufficient conditions for $\beta-$ mixing 
coefficients to decay at a polynomial rate. These conditions are expressed as restrictions on how slow the pull measure goes to zero at one of the boundaries. For some of these strongly dependent processes we may find functions of the Markov state with spectral densities that are infinite at frequency zero. Thus in spite of the first-order Markov property, best linear predictors are compelled to weight heavily past observations. We display scalar diffusions whose spectral densities near frequency zero diverge in the same manner as stationary linear models that are fractionally integrated. ${ }^{1}$

Our findings complement the work of Granger and Teräsvirta (1999), who produce a (discrete-time) nonlinear Markov model with sample statistics that suggest evidence for long memory. We construct Markov models for which population spectral densities diverge because of the forms of the nonlinearities in the conditional means and the conditional volatilities. Our construction differs from the nonlinear example in Granger and Teräsvirta (1999), since theirs is still weakly dependent as measured by the population attributes. It only looks like a long memory process from the vantage point of sample statistics. ${ }^{2}$ In contrast, the first-order strictly stationary Markov examples we construct are strongly dependent in the population.

We conclude this paper by studying how the temporal dependence of a diffusion is altered when it is sampled in a state dependent manner. For convenience, we use the Poisson sampling scheme advocated by Duffie and Glynn (2004) with a state dependent intensity parameter. The state dependence and hence endogeneity in the sampling alters the temporal dependence and the stationary distribution. We show how to adjust the measure of pull to take account of this endogeneity.

The rest of the paper is organized as follows. Section 2 briefly reviews the concepts of $\rho-$ mixing and $\beta-$ mixing for a continuous-time stationary Markov process. Section 3 establishes that $\rho$-mixing is equivalent to exponential ergodicity for a scalar diffusion, which in turn implies $\beta$ - mixing with exponential decay. Section 4 provides sufficient conditions for $\rho-, \beta-$ mixing with exponential decay rates expressed as boundary pull restrictions. Sections 5 and 6 study strongly dependent diffusion processes in the sense that $\rho_{t} \equiv 1$ for all $t \geq 0$, and $\beta$-mixing with sub-exponential decay rates. While Section 5 provides sufficient conditions for $\beta$ - mixing with sub-exponential decay rates, Section 6 presents examples where some nonlinear transformations of the strongly dependent Markov diffusions behave as long memory processes. Section 7 considers the diffusions subject to a Poisson sampling. Section 8 concludes.

\footnotetext{
${ }^{1}$ While we provide simple sufficient conditions for $\beta-$ mixing decay rates in terms of the pull measures, there are alternative sufficient conditions for a diffusion process to be $\rho-$ mixing or $\beta$ - mixing; see, e.g., Genon-Catalot, Jeantheau, and Laredo (2000) for $\rho$ - mixing and Veretennikov (1987) and Veretennikov (1997) for $\beta$-mixing. The sufficient conditions and proof strategies in these papers are different from ours. More importantly, they do not discuss the possibility of generating long memory type behavior from strongly dependent strictly stationary Markov diffusions.

${ }^{2}$ Similarly, Diebold and Inoue (2001) present nonlinear time series models, including regime switching, structure change, stochastic permanent break models, which look like long memory models in terms of growth rate of sample variance of partial sums. Hidalgo and Robinson (1996) discuss the difficulty of detecting a structural break model from a long memory model.
} 


\section{Review: Mixing Conditions}

Consider a stationary Markov process $\left\{x_{t}\right\}$ on an open connected set $\Omega \subseteq \mathbb{R}^{n}$. For convenience, use a stationary distribution $Q$ to initialize this process. Let $L^{2}$ denote the space of Borel measurable functions mapping $\Omega \rightarrow \mathbb{R}$ that are square integrable against the stationary distribution $Q$. Associated with the Markov process $\left\{x_{t}: t \geq 0\right\}$ is a semigroup of conditional expectation operators $\left\{\mathcal{T}_{t}: t \geq 0\right\}$ defined on $L^{2}$ :

$$
\mathcal{T}_{t} \phi(x)=E\left[\phi\left(x_{t}\right) \mid x_{0}=x\right]
$$

For notational simplicity, we also let $\mathcal{T}$ be shorthand notation for $\mathcal{T}_{1}$. As in Hansen and Scheinkman (1995), we suppose that the semigroup is (right) continuous at $t=0$. This allows us to construct a generator $\mathcal{A}$ on a domain $D$ that is dense in $L^{2}$ :

$$
\mathcal{A} \phi(x)=\lim _{t \searrow 0} \frac{E\left[\phi\left(x_{t}\right) \mid x_{0}=x\right]-\phi(x)}{t}
$$

where the limit is defined using the mean-square norm on $L^{2}$. Thus the generator is the time zero derivative of the semigroup of conditional expectation operators.

\section{$2.1 \quad \rho$ - mixing}

Our first notion of mixing uses functions on the ball surface:

$$
U=\left\{\phi: \int \phi d Q=0,\|\phi\|_{2}=1\right\},
$$

where $\|\cdot\|_{2}$ denotes the mean-square norm on $L^{2}$.

Definition 2.1. (Rosenblatt (1971)) The $\rho$ - mixing coefficients are given by:

$$
\rho_{t}=\sup _{\psi, \phi \in U} \int \psi\left(\mathcal{T}_{t} \phi\right) d Q .
$$

The process $\left\{x_{t}\right\}$ is $\rho-$ mixing if $\lim _{t \rightarrow \infty} \rho_{t}=0$; and is $\rho-$ mixing with exponential decay rate if $\rho_{t} \leq \exp (-\delta t)$ for some $\delta>0$.

Banon (1977) (Lemma 3.11) and Bradley (1986) (Theorem 4.2) established that for a stationary Markov process either the $\rho$-mixing coefficients decay exponentially or they are identically equal to one.

\section{$2.2 \beta$ - mixing}

An alternative way of measuring temporal dependence is given by the $\beta$ - mixing coefficients. $^{3}$

\footnotetext{
${ }^{3} \beta-$ mixing is also called absolutely regular. It was studied by Volkonskii and Rozanov (1959), but they attribute the concept to Kolmogorov. The definition presented here is an alternative but equivalent one for a stationary Markov process, see e.g. Davydov (1973).
} 
Definition 2.2. (Davydov (1973)) The $\beta$ - mixing coefficients are given by:

$$
\beta_{t}=\int \sup _{0 \leq \phi \leq 1}\left|\mathcal{T}_{t} \phi(x)-\int \phi d Q\right| d Q
$$

The process $\left\{x_{t}\right\}$ is $\beta$ - mixing if $\lim _{t \rightarrow \infty} \beta_{t}=0$; is $\beta$ - mixing with exponential decay rate if $\beta_{t} \leq \gamma \exp (-\delta t)$ for some $\delta, \gamma>0$; and is $\beta$ - mixing with sub-exponential decay rate if $\lim _{t \rightarrow \infty} \xi_{t} \beta_{t}=0$ for some positive non-decreasing rate function $\xi$ satisfying $\xi_{t} \rightarrow \infty$, $t^{-1} \ln \xi_{t} \rightarrow 0$ as $t \rightarrow \infty$.

In contrast to the $\rho$-mixing coefficients, the $\beta$ - mixing coefficients need not to converge to zero at an exponential rate. In fact, any positive (Harris) recurrent, aperiodic Markov process is $\beta$ - mixing; see Davydov (1973) (Corollary) and Bradley (1986) (Theorem 4.3). For general stationary Markov processes, the two dependence measures are not comparable: $\rho-$ mixing does not imply $\beta-$ mixing and $\beta-$ mixing does not imply $\rho-$ mixing. ${ }^{4}$ Nevertheless, all of the diffusion models we consider in this paper are $\beta$-mixing, but some have $\rho_{t} \equiv 1$ for all $t$.

\section{$2.3 f$ - ergodicity}

The notion $\beta$ - mixing for a Markov process is closely related to the concept called $f-$ ergodicity (in particular 1 - ergodicity), see e.g., Meyn and Tweedie (1993).

Definition 2.3. Given a Borel measurable function $f \geq 1$, the Markov process $\left\{x_{t}\right\}$ is $f$-ergodic if

$$
\lim _{t \rightarrow \infty} \sup _{0 \leq \phi \leq f}\left|\mathcal{T}_{t} \phi(x)-\int \phi d Q\right|=0, \text { for all } x
$$

The Markov process $\left\{x_{t}\right\}$ is $f$-uniformly ergodic if for all $t \geq 0$,

$$
\sup _{0 \leq \phi \leq f}\left|\mathcal{T}_{t} \phi(x)-\int \phi d Q\right| \leq c f(x) \exp (-\delta t)
$$

for positive constants $c$ and $\delta .^{5}$

A stationary process that is f-uniformly ergodic will be $\beta$ - mixing with exponential decay rate provided that $E f\left(x_{t}\right)<\infty$. This connection is valuable because Meyn and Tweedie (1993) and Down, Meyn, and Tweedie (1995) provide convenient drift conditions for $f$-uniform ergodicity. There exist other methods to establish 1 -ergodicity (i.e. when $f \equiv 1$ ) with sub-exponential decay rates, for example see Lindvall (1983).

\footnotetext{
${ }^{4}$ See Bradley (1986) (Example 4.4) for a stationary Markov chain that is $\rho-$ mixing but $\beta_{t} \equiv 1$ for all $t$.

${ }^{5}$ Our use of the term uniform ergodicity follows Down, Meyn, and Tweedie (1995), but it differs from the use in Meyn and Tweedie (1993). Meyn and Tweedie (1993) define $f$-uniform ergodicity by requiring the left-hand side to converge to zero uniformly in $x$ as $t$ gets large.
} 


\section{Temporal dependence of a scalar diffusion}

A scalar diffusion is typically represented as the solution to a stochastic differential equation:

$$
d x_{t}=\mu\left(x_{t}\right) d t+\sigma\left(x_{t}\right) d W_{t}
$$

with left boundary $\ell$ and right boundary $r$, either of which can be infinite. The function $\mu$ is the drift, $\sigma^{2}$ is the diffusion coefficient and $\left\{W_{t}\right\}$ is a standard Brownian motion.

Assumption 3.1. $\mu$ and $\sigma$ are continuous on $(\ell, r)$ with $\sigma$ strictly positive on this interval.

The generator of this scalar diffusion is known to be the differential operator:

$$
\mathcal{A} \phi=\mu \phi^{\prime}+\frac{1}{2} \sigma^{2} \phi^{\prime \prime}
$$

We will give a precise statement of the domain $D$ of this generator subsequently.

The boundary behavior of a diffusion is characterized by the behavior of its scale function $S($.$) :$

$$
\begin{aligned}
S(x) & =\int_{a}^{x} s(y) d y, \text { for some fixed } a \in(\ell, r) \\
s(y) & =\exp \left(-\int_{a}^{y} \frac{2 \mu(u)}{\sigma^{2}(u)} d u\right) .
\end{aligned}
$$

Since the scale function is increasing, it is well defined at both boundaries. A boundary is attracting when the scale function is finite at that boundary. We focus exclusively on the case in which neither boundary is attracting:

Assumption 3.2. $S(\ell)=-\infty$ and $S(r)=+\infty$.

We provide sufficient conditions that, among other things, guarantee that there exists a stationary distribution $Q$ for the scalar diffusion. Since both boundaries are not attracting, this distribution, when it exists, is known to be unique and have a density $q$ that is proportional to $\frac{1}{s \sigma^{2}}$. Thus stationarity is satisfied when: ${ }^{6}$

Assumption 3.3. $\int_{\ell}^{r} \frac{1}{s(x) \sigma^{2}(x)} d x<\infty$.

From Has'minskii (1980) (Example 2, page 105), the process $\left\{x_{t}\right\}$ is Harris recurrent if Assumptions 3.1 and 3.2 hold. Hence $\left\{x_{t}\right\}$ is positive (Harris) recurrent if Assumptions 3.1, 3.2 and 3.3 hold. The diffusion is also aperiodic under these assumptions. Therefore,

Remark 3.4. Under Assumptions 3.1, 3.2 and 3.3, the process $\left\{x_{t}\right\}$ is $\beta$ - mixing.

Hansen and Scheinkman (1995) provide sufficient conditions for a scalar diffusion to be $\rho-$ mixing with exponential decay, and Hansen, Scheinkman, and Touzi (1998) provide alternative conditions for a diffusion to be strongly dependent in the sense that $\rho_{t} \equiv 1$ for all $t$.

\footnotetext{
${ }^{6}$ An alternative way to represent a scalar diffusion is to start with the pair $\left(Q, \sigma^{2}\right)$, see e.g. Hansen, Scheinkman, and Touzi (1998) and Chen, Hansen, and Scheinkman (2000) for such kind of approach. The advantage of this representation is that any probability measure $Q$ (with a continuous density) can be the invariant measure of a scalar diffusion.
} 


\subsection{Diffusion in natural scale}

For convenience, we transform the diffusion $\left\{x_{t}\right\}$ monotonically to its natural scale process $\left\{z_{t}\right\}$ :

$$
z_{t}=S\left(x_{t}\right)
$$

Clearly the state space for $\left\{z_{t}\right\}$ is the entire real line $(-\infty,+\infty)$ under Assumption 3.2. Moreover, $\left\{z_{t}\right\}$ in the natural scale is known to be a local martingale (have zero drift) with diffusion coefficient:

$$
\theta^{2}(z)=s^{2}\left[S^{-1}(z)\right] \sigma^{2}\left[S^{-1}(z)\right] .
$$

An equivalent statement of Assumption 3.3 is:

$$
\int_{-\infty}^{+\infty} \frac{1}{\theta^{2}}<\infty .
$$

For the natural scale diffusion, the generator is the second-order differential operator

$$
\mathcal{A} \phi=\frac{1}{2} \theta^{2} \phi^{\prime \prime}
$$

defined on the domain:

$$
D=\left\{\phi: \phi^{\prime} \text { is absolutely continuous and } \begin{array}{l}
\int \frac{1}{\theta^{2}} \phi^{2}<\infty \\
\left.\int \phi^{\prime}\right)^{2}<\infty \\
\int \theta^{2}\left(\phi^{\prime \prime}\right)^{2}<\infty
\end{array}\right\} .
$$

\subsection{Equivalence of $f$-uniform ergodicity to $\rho$-mixing}

We already pointed out that $f$-uniform ergodicity implies $\beta$ - mixing with exponential decay. In this section, we explore further the near equivalence of $f$-uniform ergodicity and $\rho$ - mixing for a stationary scalar diffusion. To establish such an equivalence, we use a characterization of $f$ - uniform ergodicity by Down, Meyn, and Tweedie (1995) and a characterization of $\rho$-mixing by Hansen, Scheinkman, and Touzi (1998).

Let $C^{2}$ denote the space of functions mapping $(\ell, r)$ into $\mathbb{R}$ with continuous first and second derivatives. We construct the local operator:

$$
\mathcal{B} V=\mu V^{\prime}+\frac{1}{2} \sigma^{2} V^{\prime \prime}
$$

which coincides with the generator $\mathcal{A}$ on the intersection of the domain $D$ of the generator and $C^{2}$. A non-negative function $V$ in $C^{2}$ is norm-like if $\{x: V(x) \leq v\}$ is compact in $(\ell, r)$ for any $v>0$.

The following lemma is Theorem 5.2 of Down, Meyn, and Tweedie (1995) (page 1681) specialized to our scalar diffusion $\left\{x_{t}\right\}$ :

Lemma 3.5. Suppose Assumptions 3.1 and 3.2 hold, and that there exists a non-negative function $V \in C^{2}$ (not necessarily norm-like) such that:

$$
\mathcal{B} V \leq-c(V+1)+d \mathbf{1}_{K}
$$

for some positive constants $c$ and $d$ and some compact set $K$. Then: (i) $\left\{x_{t}\right\}$ has a unique invariant probability measure $Q$; (ii) $\int V(x) d Q(x)<\infty$; (iii) $\left\{x_{t}\right\}$ is $(V+1)$-uniformly ergodic. 
For a diffusion in natural scale, we consider solutions to the second-order differential equation:

$$
\mathcal{B} \phi=-c \phi
$$

for $\phi \in C^{2}$, and some $c>0$, which is in the form of an eigenvalue problem. Weidmann (1987) (page 225) shows that there exists a nonnegative $a$ such that for $c \geq a$ solutions $\phi$ cross the zero axis only a finite number of times and for $c<a$ they cross the axis an infinite number of times. Hansen, Scheinkman, and Touzi (1998) show that the corresponding diffusion is $\rho$ - mixing if, and only if $a>0$. We exploit this characterization of $\rho$-mixing to establish the following two theorems.

Theorem 3.6. Suppose that Assumptions 3.1, 3.2 and 3.3 hold, and $\left\{x_{t}\right\}$ is $\rho-$ mixing. Then: there exists a nonnegative function $V \in C^{2}$ such that

$$
\frac{1}{2} \theta^{2} V^{\prime \prime} \leq-c(V+1)+d \mathbf{1}_{K}
$$

for some compact interval $K$ and positive constants $c$ and $d$. In particular, the diffusion is $(V+1)$ uniformly ergodic and $\beta$ - mixing with exponential decay.

Proof. See Appendix.

We next present a (partial) converse to this result.

Theorem 3.7. Suppose that Assumptions 3.1 and 3.2 hold, and there is a nonnegative function $V \in C^{2}$, a compact interval $K$ and positive constants $c$ and $d$ such that

$$
\frac{1}{2} \theta^{2} V^{\prime \prime} \leq-c(V+1)+d \mathbf{1}_{K}
$$

Then: $\left\{x_{t}\right\}$ satisfies Assumption 3.3 and is $\rho$-mixing.

Proof. See Appendix.

\section{Mixing with exponential decay rates}

In this section we show that

$$
\frac{\mu}{\sigma}-\frac{\sigma^{\prime}}{2}
$$

provides a measure of the pull of the diffusion by establishing formally its implications for weak dependence (i.e., $\rho-, \beta$-mixing with exponential decay rates). We also propose extensions of this measure that avoid differentiability of the diffusion coefficient.

There are two ways to establish mixing with exponential decay. One is to obtain sufficient conditions by applying Theorem 3.7. An alternative is to apply the following lemma, which is based on Theorems 3.2, 4.4 and 6.1 of Meyn and Tweedie (1993) (pages 528, 531 and 536): ${ }^{7}$

\footnotetext{
${ }^{7}$ Meyn and Tweedie (1993)'s Theorem 6.1 requires: (1) $\left\{x_{t}\right\}$ is a right process; and (2) all compact subsets of $\Omega$ are petite for some skeleton chain. Both are satisfied given our Assumption 3.1.
} 
Lemma 4.1. Suppose Assumption 3.1 holds, and that there exists a norm-like function $V$ such that:

$$
\mathcal{B} V \leq-c V+d
$$

for some constants $c$ and $d$. Then: (i) $\left\{x_{t}\right\}$ is recurrent; (ii) If in addition $c>0$, then $\left\{x_{t}\right\}$ has a unique invariant probability measure $Q$, and $\int V(y) Q(d y)<\infty$. Moreover, $\left\{x_{t}\right\}$ is $(V+1)$-uniformly ergodic.

Both Theorem 3.7 and Lemma 4.1 can be used to establish $(V+1)$-uniform ergodicity and mixing with exponential decay. Inequality (4) of Lemma 4.1 is stronger than inequality (3) (or 2) of Theorem 3.7 since $V$ is required to be norm-like in inequality (4). On the other hand, inequality (4) implies Assumption 3.2.

\subsection{Natural Scale Case}

We first study the temporal dependence of diffusions in the natural scale. As we will see, for these diffusions there is a direct link between the thickness of the tails of stationary density and the temporal dependence of the diffusion. We use this link to provide convenient sufficient conditions for decay rates of the mixing coefficients.

Theorem 4.2. Suppose Assumption 3.1 and the following inequality hold:

$$
\liminf _{|z| \rightarrow \infty} \frac{\theta(z)}{|z|}>0
$$

Then: (i) Assumptions 3.2 and 3.3 are satisfied; (ii) $\left\{z_{t}\right\}$ is $\rho$ - and $\beta$ - mixing with exponential decay.

Proof. We establish this result by applying Lemma 4.1 and Theorems 3.6 and 3.7. We consider the following Lyapunov function:

$$
V(z)=\frac{|z|^{\alpha}}{\alpha},|z| \geq 1
$$

for some $0<\alpha<1$, and we fill in the function $V$ on $(-1,1)$ so that it is $C^{2}$ and nonnegative. The constructed function is norm-like, and as prescribed by inequality (4) we restrict $\theta^{2}$ to satisfy:

$$
\frac{\theta^{2}}{2} V^{\prime \prime} \leq-c V+d, c>0
$$

for positive constants $c$ and $d$. Inequality (6) (i.e. inequality (4)) will be satisfied if we can find positive constants $c$ and $d$ such that

$$
\frac{1}{2}(\alpha-1) \theta^{2}(z)|z|^{\alpha-2} \leq-c \frac{|z|^{\alpha}}{\alpha}+d \text { for }|z| \geq 1
$$

If necessary, we adjust the constant $d$ so that (6) is satisfied over the entire real line. Such constants exist given inequality (5). Clearly this condition is also sufficient for

$$
\int \frac{1}{\theta^{2}}<\infty
$$

which guarantees the existence of a stationary distribution (Assumption 3.3). 


\subsection{Diffusion with Nonzero Drift}

If a diffusion $\left\{z_{t}\right\}$ in natural scale is stationary and $\beta$-mixing with exponential decay rates, clearly so is the original process $\left\{x_{t}\right\}$ where $x_{t}=S\left(z_{t}\right)$. Transforming the limit in (5) of Theorem 4.2 back to the original scale, we obtain:

Corollary 4.3. Suppose that Assumptions 3.1 and 3.2 are satisfied. If

$$
\begin{aligned}
& \lim \inf _{x / r} \frac{s \sigma}{S}>0 \\
& \lim \sup _{x \searrow \ell} \frac{s \sigma}{S}<0,
\end{aligned}
$$

then: $\left\{x_{t}\right\}$ satisfies Assumption 3.3, and is $\rho$ - and $\beta$ - mixing with exponential decay.

Remark 4.4. Under Assumptions 3.1 and 3.2, if $\sigma$ is differentiable, then the inequalities in Corollary 4.3 are implied by:

$$
\begin{aligned}
& \limsup _{x \operatorname{su}^{\prime}}\left(\frac{\mu}{\sigma}-\frac{\sigma^{\prime}}{2}\right)<0 \\
& \lim \inf _{x \searrow \ell}\left(\frac{\mu}{\sigma}-\frac{\sigma^{\prime}}{2}\right)>0
\end{aligned}
$$

We may think of

$$
\frac{\mu}{\sigma}-\frac{\sigma^{\prime}}{2}
$$

as providing a measure of the pull of the diffusion. For the scalar diffusion to be $\beta$-mixing with exponential decay we require that the pull be negative at the right boundary and positive at the left boundary. These restrictions are identical to the ones proposed by Hansen and Scheinkman (1995) for $\rho$-mixing with exponential decay, albeit their derivation is different than ours.

Remark 4.5. Instead of assuming the differentiability of $\sigma$, we can require the existence of a positive function $g$ that is differentiable and is dominated by $\sigma$ :

$$
\frac{\sigma}{g} \geq 1
$$

The inequalities in Corollary 4.3 are now implied by:

$$
\begin{array}{r}
\lim \sup _{x \nearrow^{\prime}}\left(\frac{\mu}{\sigma^{2}} g-\frac{g^{\prime}}{2}\right)<0 \\
\lim \inf _{x \searrow \ell}\left(\frac{\mu}{\sigma^{2}} g-\frac{g^{\prime}}{2}\right)>0 .
\end{array}
$$

Notice that these inequalities provide a trade-off between the drift and the diffusion behavior and cover examples in which the drift is dominated by the square root of the diffusion coefficient. In these cases, the exponential decay of the $\beta$-mixing coefficients is induced by the rapid increase in the volatility as a function of the Markov state. The drift may even be positive for states in the vicinity of the right boundary and negative in the 
vicinity of the left boundary (suggesting a pull to the left) while the resulting diffusion may still be stationary and uniformly ergodic. (See Conley, Hansen, Luttmer, and Scheinkman (1997) for a further discussion of volatility-induced stationarity.) Also, these conditions permit the diffusion coefficient to go to zero at either boundary.

We conclude this section by relating our work to that of Veretennikov (1987). Notice that for the special case when (i) $\sigma$ is differentiable, we can transform our original process (1) using the twice differentiable function:

$$
R(x)=\int_{d}^{x} \frac{1}{\sigma(u)} d u,
$$

where $d$ is an interior point in the state space $(\ell, r)$. This scale transformation results in a new diffusion process $\left\{y_{t}=R\left(x_{t}\right): t \geq 0\right\}$ with state space $(R(\ell), R(r))$, a unit diffusion coefficient $a^{2}=1$ and a drift given by our pull measure:

$$
b=\frac{\mu}{\sigma}-\frac{\sigma^{\prime}}{2} .
$$

Under an additional condition (ii) $(R(\ell), R(r))=(-\infty, \infty),{ }^{8}$ one can now apply Veretennikov (1987)'s theorem to establish $\beta$ - mixing with exponential decay based on the behavior of the drift coefficient $b$ of the transformed diffusion with a constant diffusion coefficient. While this provides an alternative way to justify our (7) as a sufficient condition for $\beta$ - mixing with exponential decay, our derivation does not need the extra conditions (i) and (ii). ${ }^{9}$

\section{$5 \quad \beta$-Mixing with Polynomial Decay Rates}

Previously, we deduced sufficient condition for a diffusion process to be weakly dependent. In this section, we study diffusions which are strongly dependent in the sense that the $\rho-$ mixing coefficients $\rho_{t} \equiv 1$ for all $t \geq 0$, or $\beta-$ mixing coefficients decay at rates that are slower than exponential.

To study strong dependence, we focus on cases in which the pull measure

$$
\frac{\mu}{\sigma}-\frac{\sigma^{\prime}}{2}
$$

converges to zero at one of the two boundaries. To characterize the strong dependence we study how fast this measure converges to zero. This leads us to investigate the limits:

$$
\begin{aligned}
& \nu^{+} \equiv \limsup _{x / r}\left[\left(\frac{\sigma^{2}}{\sigma \sigma^{\prime}-2 \mu}\right)^{\prime}-\frac{2 \mu}{\sigma \sigma^{\prime}-2 \mu}\right] \\
& \nu^{-} \equiv \liminf _{x \searrow \ell}\left[\left(\frac{\sigma^{2}}{\sigma \sigma^{\prime}-2 \mu}\right)^{\prime}-\frac{2 \mu}{\sigma \sigma^{\prime}-2 \mu}\right]
\end{aligned}
$$

to obtain a more refined measure of strong dependence. These limits bound the behavior of the $\beta$-mixing coefficients.

\footnotetext{
${ }^{8}$ Notice that for volatility-induced stationarity type of diffusion models, it may well be $R(\ell)>-\infty$ and/or $R(r)<\infty$. So $(R(\ell), R(r))=(-\infty, \infty)$ is an additional restriction.

${ }^{9}$ In particular, our Corollary 4.3 cannot be derived from Veretennikov (1987)'s theorem.
} 


\subsection{Hitting Times}

For a general discrete time Markov process, the $\beta$-mixing polynomial decay rates are implied by restrictions on the moments of the random time it takes to hit a compact set; see e.g., Tuominen and Tweedie (1994) (theorems 2.3 and 4.3). A version of this kind of result for scalar diffusion was established by Lindvall (1983), which we now state. ${ }^{10}$ Let $\tau_{K}$ denote the first time that the process $\left\{x_{t}\right\}$ hits the point $K$ in the interior of the state space:

$$
\tau_{K}=\inf \left\{t \geq 0, x_{t}=K\right\} .
$$

Theorem 5.1. Suppose Assumptions 3.1, 3.2 and 3.3 hold. Suppose that there is a function $\xi \geq 0$ non-decreasing on $[0, \infty)$ such that $E\left(\xi\left(\tau_{K}\right)\right)<\infty$. Then

$$
\lim _{t \uparrow \infty} \xi(t) \beta_{t}=0
$$

if further, $\xi()$ is absolutely continuous with respect to Lebesgue measure and has a density $(\xi)^{\prime}$, then

$$
\int_{0}^{\infty}(\xi(t))^{\prime} \beta_{t}<\infty .
$$

In particular, if $\xi(t)=t^{\delta}$ for some $\delta>0$ and $E\left(\tau_{K}\right)^{\delta}<\infty$, then:

$$
\begin{gathered}
\int_{0}^{\infty} t^{\delta-1} \beta_{t}<\infty \\
\lim _{t \rightarrow \infty} t^{\delta} \beta_{t}=0 .
\end{gathered}
$$

Proof. See Appendix.

\subsection{Natural Scale Case}

One strategy for establishing the hitting time moment bounds of Theorem 5.1 is to follow Lindvall (1983) and study natural scale diffusion processes. Transforming the scale does not alter the hitting time distribution. Lindvall (1983) derives a sufficient condition for $E\left(\tau_{K}\right)^{\delta}<\infty$ based on a moment restriction expressed using the natural scale stationary distribution. The following result is based on lemma 1 and proposition 2 of Lindvall (1983) and our Theorem 5.1, but is stated in terms of growth rates of $\theta$. It is the counterpart of Theorem 4.2, but for slower growth rates.

Theorem 5.2. Suppose that Assumptions 3.1 and 3.2 hold, and there exists some constant $\frac{1}{2}<\eta<1$ such that:

$$
\liminf _{|z| \rightarrow \infty} \frac{\theta(z)}{|z|^{\eta}}>0 .
$$

Let

$$
\eta^{*} \equiv \sup \{\eta: \text { inequality (9) is satisfied }\} .
$$

Then: (i) Assumption 3.3 is satisfied; (ii) $E\left(\tau_{K}\right)^{\delta}<\infty$ for any $\delta<\delta^{*}$ where $\delta^{*}=\frac{2 \eta^{*}-1}{2-2 \eta^{*}}$. In particular, $\left\{z_{t}: t \geq 0\right\}$ is $\beta-$ mixing and for any $\delta<\delta^{*}, \lim _{t \rightarrow \infty} t^{\delta} \beta_{t}=0$.

\footnotetext{
${ }^{10}$ Lindvall (1983) does not make any link to $\beta$-mixing, but, as we show in the appendix, it is easy to modify his result to obtain $\beta$-mixing coefficients decaying at a polynomial order.
} 
Proof. Equation (9) implies the result (i) as long as $\eta>1 / 2$. Equation (9) also implies that

$$
\int_{-\infty}^{\infty}|z|^{1-(\alpha)^{-1}} \frac{1}{\theta^{2}(z)} d z<\infty \text { provided } 2 \eta+(\alpha)^{-1}-1>1
$$

Let $0<\delta \equiv \alpha-1<\frac{2 \eta-1}{2-2 \eta}$. Then by lemma 1 or proposition 2 of Lindvall (1983), we have $E\left(\tau_{K}\right)^{\delta}<\infty$. The result (ii) now follows from Theorem 5.1 inequality (8).

Theorem 5.2 gives interesting results when the tail growth of $\theta$ as a function of $|z|$ exceeds $|z|^{\frac{1}{2}}$ but is less than linear. Slower growth in $\theta$ implies slower decay in the $\beta-$ mixing coefficients.

We next derive a sufficient condition for (9) in the natural scale. Although we derive this in terms of the natural scale, our interest in this sufficient condition is its counterpart in the original scale. The logarithmic derivative of a power function is proportional to $1 / z$. The coefficient used in this proportionality dictates when the tail inequality (9) is satisfied. Thus we are led to compute the derivative:

$$
\left(\frac{\theta}{\theta^{\prime}}\right)^{\prime}=1-\frac{\theta^{\prime \prime}(z) \theta(z)}{\theta^{\prime}(z)^{2}}
$$

to study the tail behavior of $\theta$. We are interested in this limit in the case in which $\theta^{\prime}$ tends to zero. Define,

$$
\begin{aligned}
& \nu^{+} \equiv \limsup _{z \rightarrow+\infty}\left[1-\frac{\theta^{\prime \prime}(z) \theta(z)}{\theta^{\prime}(z)^{2}}\right] \\
& \nu^{-} \equiv \liminf _{z \rightarrow-\infty}\left[1-\frac{\theta^{\prime \prime}(z) \theta(z)}{\theta^{\prime}(z)^{2}}\right]
\end{aligned}
$$

Moreover, let

$$
\nu^{*} \equiv\left\{\begin{array}{ccc}
\nu^{+} & \text {if } & \lim \sup _{z \searrow-\infty} \theta^{\prime}(z)>0 \\
\nu^{-} & \text {if } & \liminf \inf _{z \nearrow+\infty} \theta^{\prime}(z)<0 \\
\max \left\{\nu^{+}, \nu^{-}\right\} & & \text {otherwise. }
\end{array}\right.
$$

Lemma 5.3. Suppose that $\theta$ is twice differentiable, and that

$$
\begin{array}{r}
\liminf _{z \nearrow+\infty} \theta^{\prime}(z) \leq 0, \\
\limsup _{z \backslash-\infty} \theta^{\prime}(z) \geq 0,
\end{array}
$$

where at least one of these two limits is zero. If $1<\nu^{*}<2$, then (9) is satisfied for any $\eta<\frac{1}{\nu^{*}}$. In particular, $\eta^{*} \geq \frac{1}{\nu^{*}}$.

Proof. We prove this result when both limiting derivatives are equal to zero. The other cases can be proved using a more direct argument for one boundary and an entirely similar argument to what follows for the other boundary. Consider any $\frac{1}{\eta}>\nu^{*}$. For sufficiently large $z^{*}$, and $z \geq z^{*}$,

$$
\frac{1}{(\log \theta)^{\prime}(z)} \leq \frac{z}{\eta}+c_{1}
$$


and $z \leq-z^{*}$

$$
\frac{1}{(\log \theta)^{\prime}(z)} \geq \frac{z}{\eta}-c_{1}
$$

where $c^{+}$and $c^{-}$are some appropriately chosen positive constants. Taking reciprocals reverses the inequalities. Hence taking reciprocals and integrating implies that for $z \geq z^{*}$ :

$$
\log \theta(z) \geq \eta \log \left(z / \eta+c_{1}\right)+c_{2}
$$

and for $z \leq-z^{*}$ :

$$
\log \theta(z) \geq \eta \log \left(-z / \eta+c_{1}\right)+c_{2}
$$

for some appropriately chosen constant $c_{2}$. Consequently, we may find a positive constant $c_{3}$ such that

$$
\theta(z) \geq c_{3}\left(|z| / \eta+c_{1}\right)^{\eta}
$$

for $|z| \geq z^{*}$. Therefore, (9) is satisfied.

The following example illustrates the polynomial bounds on the $\beta$-mixing coefficients and in particular when Lemma 5.3 produces the same bounds $\left(\right.$ i.e. $\left.\eta^{*}=\frac{1}{\nu^{*}}\right)$.

Example 5.4. Suppose that $\theta(z)=\left(1+|z|^{2}\right)^{\frac{\gamma}{2}}$ and $1 / 2<\gamma<1$. It may be shown directly that $\eta^{*}=\gamma$. Thus from Theorem 5.2, $\lim _{t \rightarrow \infty} t^{\delta} \beta_{t}=0$ for any

$$
\delta<\frac{2 \gamma-1}{2-2 \gamma}
$$

Notice also that $\nu^{*}$ used in Lemma 5.3 is given by $\nu^{*}=\frac{1}{\gamma}=\frac{1}{\eta^{*}}$.

\subsection{Diffusion with a Nonzero Drift}

Our main interest in Lemma 5.3 is its implication for a diffusion process in the original scale. We now transform the condition given in this lemma. Note that

$$
\theta^{\prime}=-2\left(\frac{\mu}{\sigma}-\frac{\sigma^{\prime}}{2}\right)=\left(\frac{\sigma \sigma^{\prime}-2 \mu}{\sigma}\right) .
$$

We are interested in cases in which $\theta^{\prime}$ tends to zero. Since $\theta=s \sigma$,

$$
\left(\frac{\theta}{\theta^{\prime}}\right)^{\prime}=\frac{1}{s}\left(\frac{s \sigma^{2}}{\sigma \sigma^{\prime}-2 \mu}\right)^{\prime} .
$$

where the term $1 / s$ on the right-hand side is included because of the change in variables when computing the derivative. This correction is needed because the derivative on the left-hand side is with respect to the natural scale and the derivative on the right-hand side is with respect to the original scale. The right-hand derivative can be written equivalently without reference to the scale density $s$ as

$$
\left(\frac{\theta}{\theta^{\prime}}\right)^{\prime}=\left(\frac{\sigma^{2}}{\sigma \sigma^{\prime}-2 \mu}\right)^{\prime}-\frac{2 \mu}{\sigma \sigma^{\prime}-2 \mu} .
$$


This leads us to define:

$$
\begin{aligned}
& \nu^{+} \equiv \limsup _{x / r}\left[\left(\frac{\sigma^{2}}{\sigma \sigma^{\prime}-2 \mu}\right)^{\prime}-\frac{2 \mu}{\sigma \sigma^{\prime}-2 \mu}\right] \\
& \nu^{-} \equiv \liminf _{x \searrow \ell}\left[\left(\frac{\sigma^{2}}{\sigma \sigma^{\prime}-2 \mu}\right)^{\prime}-\frac{2 \mu}{\sigma \sigma^{\prime}-2 \mu}\right]
\end{aligned}
$$

Corollary 5.5. Suppose that

$$
\begin{aligned}
& \limsup _{x \nearrow r}\left(\frac{\mu}{\sigma}-\frac{\sigma^{\prime}}{2}\right) \leq 0 \\
& \liminf _{x \backslash \ell}\left(\frac{\mu}{\sigma}-\frac{\sigma^{\prime}}{2}\right) \geq 0
\end{aligned}
$$

with at least one of these limits equal to zero. Let $\nu^{*}$ be $\nu^{+}$if only the first limit is zero, be $\nu^{-}$if only the second limit is zero, and be $\max \left\{\nu^{+}, \nu^{-}\right\}$if both limits are zero. If $1<\nu^{*}<2$, then for any $\delta<\frac{2-\nu^{*}}{2 \nu^{*}-2}$ the process $\left\{x_{t}: t \geq 0\right\}$ is $\beta-$ mixing and $\lim _{t \rightarrow \infty} t^{\delta} \beta_{t}=0$.

This corollary shows how to use formula (10) to compute a polynomial bound on the rate of decay of the $\beta$ mixing coefficients when the pull measure is zero in one of the two tails.

Example 5.6. Suppose that $\sigma=1$ and

$$
\mu(x)\left\{\begin{array}{l}
\leq-\frac{\kappa}{x} \quad x \geq a \\
\geq \quad-\frac{\kappa}{x} \quad x \leq-a
\end{array}\right.
$$

for some positive $\kappa$ and $a$ as in Veretennikov (1997). Then

$$
\nu^{*} \equiv 1+\frac{1}{2 \kappa} \in(1,2) \text { provided } \kappa>\frac{1}{2},
$$

and the restriction on $\delta$ is:

$$
\delta<\frac{2-\nu^{*}}{2 \nu^{*}-2}=\kappa-\frac{1}{2}
$$

This matches the conclusion in Veretennikov (1997) for a scalar diffusion.

\section{Strong Dependence and Spectral Densities}

For linear time series models, it is common to link temporal dependence to the behavior of the spectral density near frequency zero. For instance, the rate of divergence of the spectral density at frequency zero gives a way to characterize long memory of a stochastic process. For this reason, we now examine the implied behavior of spectral density function for test functions applied to the Markov diffusion. In what follows, we will first deduce a convenient formula for calculating the spectral density at a given frequency for transformations of a natural scale diffusion. Then we will construct diffusion processes with spectral densities that diverge at frequency zero. For this phenomenon to occur, at the very least we need the processes to fail being $\rho$-mixing. However, even when the $\rho$-mixing coefficients are identically one, the spectral density at frequency zero will still be finite for many (but not all) functions of the Markov state. In particular, we will use Example 5.4 as a starting point for a natural scale diffusion that fails to be $\rho-$ mixing, and transform the state space to obtain Markov processes with divergent spectral density functions. 


\subsection{A Formula for the Spectral Density}

Let $\left\{z_{t}\right\}$ be a natural scale diffusion with diffusion coefficient $\theta^{2}$ (and generator $\mathcal{A}=\theta^{2} / 2$ ). Let $Z \equiv\left\{\phi \in L^{2}: \int \phi d Q=0\right\}$ denote the class of real-valued test functions with zero means and finite variances, where $Q$ has density proportional to $\frac{1}{\theta^{2}}$. For any test function $\phi \in Z$, the process $\left\{\phi\left(z_{t}\right)\right\}$ is stationary $\beta$ - mixing, hence its spectral measure is absolute continuous and the spectral density $f(\omega)$ exists satisfying $\int_{-\infty}^{\infty} \frac{|\ln f(\omega)|}{1+\omega^{2}} d \omega<\infty$ and can be represented as: (see e.g. Ibragimov and Rozanov (1978), pages 34-36, 112 and 138)

$$
f(\omega)=\frac{1}{2 \pi} \int_{-\infty}^{+\infty} \exp (-i \omega t)\left[\int \phi\left(\mathcal{T}_{t} \phi\right) d Q\right] d t \quad \text { if } \int_{0}^{\infty}\left[\int\left(\phi \mathcal{T}_{t} \phi\right) d Q\right] d t<\infty
$$

and

$$
\begin{aligned}
f(\omega) & =\lim _{M \rightarrow \infty} f_{M}(\omega) \quad \text { in } \mathcal{L}^{2}((-\infty, \infty), L e b) \quad \text { if } \int_{0}^{\infty}\left[\int\left(\phi \mathcal{T}_{t} \phi\right) d Q\right]^{2} d t<\infty, \\
f_{M}(\omega) & =\frac{1}{2 \pi} \int_{-M}^{+M} \exp (-i \omega t)\left[\int \phi\left(\mathcal{T}_{t} \phi\right) d Q\right] d t
\end{aligned}
$$

Since the natural scale diffusion $\left\{z_{t}\right\}$ is time-reversible, the autocorrelations are nonnegative:

$$
\int\left(\phi \mathcal{T}_{t} \phi\right) d Q \geq 0
$$

Thus the spectral density function at frequency zero dominates the spectral density at all other frequencies.

For any given $\phi \in Z$ and frequency $\omega$, we solve the differential equation system:

$$
(\mathcal{A}-i \omega \mathcal{I}) \psi=\phi
$$

where $\psi=\psi_{r}+i \psi_{i}$ and $\psi_{r}, \psi_{i} \in D$, (notice that the solution $\psi$ depends implicitly on $\omega$ ). Thus $\psi$ solves the pair of differential equations:

$$
\begin{aligned}
& \frac{\theta^{2}}{2} \psi_{r}^{\prime \prime}=-\omega \psi_{i}+\phi \\
& \frac{\theta^{2}}{2} \psi_{i}^{\prime \prime}=\omega \psi_{r}
\end{aligned}
$$

for $\psi_{r}, \psi_{i} \in D$. By construction, the solution $\psi$ satisfies for any $\omega \neq 0$

$$
\psi=(\mathcal{A}-i \omega \mathcal{I})^{-1} \phi=-\int_{0}^{\infty} \mathcal{T}_{t} \exp (-i \omega t) \phi d t
$$

Then $\left\{\phi\left(z_{t}\right)\right\}$ has a finite spectral density at frequency $\omega \neq 0$ given by:

$$
\begin{aligned}
f(\omega) & =\int \phi\left[\int_{-\infty}^{+\infty}\left(\mathcal{T}_{t} \exp (-i \omega t) \phi\right) d t\right] d Q \\
& =-2 \text { real }\left(\int \phi\left[(\mathcal{A}-i \omega \mathcal{I})^{-1} \phi\right] d Q\right) \\
& =-2\left(\int \phi \psi_{r} d Q\right) .
\end{aligned}
$$


For frequency $\omega=0$, the pair of differential equations (11) becomes $\psi_{i}=0$ and $\frac{\theta^{2}}{2} \psi_{r}^{\prime \prime}=\phi$ (i.e. $\mathcal{A} \psi=\phi$ ), which has a solution $\psi=\mathcal{A}^{-1} \phi \in D$ if and only if $\phi \in Z$ belongs to the range of $\mathcal{A}$. In this case, an integration-by-parts argument leads to:

$$
f(0)=-2 \int \phi\left(\mathcal{A}^{-1} \phi\right) d Q=\frac{\int\left(\psi_{r}^{\prime}\right)^{2}}{\int \frac{1}{\theta^{2}}} .
$$

Moreover, $f(0)$ gives the asymptotic variance for the central limit approximation for $\left\{\frac{1}{\sqrt{N}} \int_{0}^{N} \phi\left(x_{t}\right) d t\right\}$ (see Bhattacharya (1982) and Hansen and Scheinkman (1995)).

Notice that when the diffusion process $\left\{z_{t}\right\}$ is $\rho$ - mixing, the range of $\mathcal{A}$ coincides with the space $Z$, hence any test function $\phi \in Z$ has finite $f(0)$ given by the formula (12). However, when the diffusion process is strongly dependent in the sense $\rho_{t} \equiv 1$ for all $t \geq 0$, the range of $\mathcal{A}$ is merely a dense subset of $Z$; hence there exist functions $\phi \in Z$ that are outside the range of $\mathcal{A}$. For all the test functions $\phi$ that belong to the range of $\mathcal{A}$, the familiar central limit approximations continue to apply to $\left\{\phi\left(x_{t}\right)\right\}$, and for which the spectral densities remain bounded in the vicinity of frequency zero. When $\phi$ is outside the range of $\mathcal{A}$, we can no longer solve the operator equation $\mathcal{A} \psi=\phi$ in $D$. Bhattacharya (1982)'s Central Limit Theorem may fail and the spectral density may diverge at frequency zero. We now construct examples for which the spectral density becomes unbounded at frequency zero.

\subsection{Divergent Spectral Densities}

Formula (12) also suggests a way to construct transformations (test functions) $\phi$ with finite variances but infinite spectral densities at frequency zero for strongly dependent processes: find $\phi$ 's with zero means and finite variances that satisfies:

$$
\frac{\theta^{2}}{2} \psi^{\prime \prime}=\phi
$$

for which the corresponding $\psi$ is outside the domain $D$, and in particular satisfies:

$$
\int\left(\psi^{\prime}\right)^{2}=+\infty
$$

We illustrate such a construction by developing further Example 5.4:

$$
\theta(z)=\left(1+z^{2}\right)^{\gamma / 2}
$$

for $\frac{1}{2}<\gamma<1$. For this range of $\gamma^{\prime}$ 's we have already argued that the $\rho$ - mixing coefficients are all one. To find a test function $\phi$ with zero mean, finite variance and infinite spectral density at frequency zero, we use the construction:

$$
\phi=\frac{\theta^{2}}{2} \psi^{\prime \prime}
$$

and find a function $\psi$ that satisfies (13) along with

$$
\int \theta^{2}\left(\psi^{\prime \prime}\right)^{2}<\infty
$$




$$
\lim _{z \rightarrow \pm \infty} \psi^{\prime}(z)=0
$$

An example of such a function is:

$$
\psi(z)^{\prime}=\left(1+z^{2}\right)^{-\eta / 2}
$$

for

$$
\gamma-\frac{1}{2}<\eta \leq \frac{1}{2}
$$

The resulting test function $\phi$ has a finite variance and a zero mean against the stationary distribution by virtue of (14) and (15). The infinite spectral density of the process $\left\{\phi\left(z_{t}\right)\right\}$ at frequency zero is suggested by (13).

The process $\left\{\phi\left(z_{t}\right)\right\}$ is itself Markov for $\frac{1}{2}<\gamma \leq \frac{3}{4}$ (since $\phi(z)$ is decreasing), and also a scalar diffusion (since $\phi \in C^{2}$ ), but with a nonzero drift. To illustrate the divergence of the spectral density, we plot the spectral density for alternative choices of $\gamma$ and $\eta$. First, we compute the spectral density functions $f(\omega)$ for frequencies $\omega$ in the vicinity of frequency zero for three values of $(\gamma, \eta):\left(.51, \frac{1}{4}\right),\left(\frac{3}{4}, \frac{1}{3}\right),\left(.99, \frac{1}{2}\right)$ in Figure 1 . The $\gamma$ values .51 and .99 were chosen because they are near the endpoints of the interval $\left(\frac{1}{2}, 1\right)$. Recall that when $\gamma$ is greater than or equal to one, the process is $\rho$-mixing with exponential decay, and when $\gamma$ is less than or equal to $\frac{1}{2}$, the process fails to be stationary. The $\gamma$ value of $\frac{3}{4}$ is chosen because the $\beta-$ mixing coefficients are integrable for $\gamma>\frac{3}{4}$, and the mean time to hit a compact set is infinite when $\gamma \leq \frac{3}{4}$. The corresponding $\eta$ 's were chosen to be close to the midpoint of the interval $\left(\gamma-\frac{1}{2}, \frac{1}{2}\right]$ in $(16) \cdot{ }^{11}$

Long memory processes including stationary versions of fractional Brownian (see Mandelbrot and Ness (1968)) have spectral densities that behave like:

$$
\log f(\omega) \approx c_{0}-c_{1} \log (\omega)
$$

in the vicinity of frequency zero for $0<c_{1}<1$. For this reason, we also depict the spectral densities in a $\log -\log$ scale in Figure 2. Notice that while the log spectral density is distinctly convex in $\log (\omega)$ for $\gamma=.99$, it is almost linear for $\gamma=.51$. This latter behavior imitates closely the spectral density of long memory time series. We also study how $\eta$ alters the slope:

$$
\text { slope }=\frac{d \log f}{d \log \omega}
$$

in Figure 3. Notice that decreasing $\eta$ increases the magnitude of the slope, but slope never exceeds one, which is the upper bound on the parameter $c_{1}$ in (17).

Remark 6.1. In the preceding example, our calculations were based primarily on the tail properties of the natural scale diffusion coefficient $\theta$ and of the function $\psi$. We are free to

\footnotetext{
${ }^{11}$ To compute the (normalized) spectral density for frequency $\omega$, we first solved the differential equation (11) numerically subject to the boundary restrictions $\psi^{\prime}\left(z^{*}\right)=\psi^{\prime}\left(-z^{*}\right)$ for a large value of $z^{*}$. We then evaluated numerically:

$$
f(\omega)=-2 \frac{\int \frac{\phi \psi_{r}}{\theta^{2}}}{\int \frac{\phi^{2}}{\theta^{2}}}
$$

and checked the sensitivity of the answer to the choice of $z^{*}$.
} 


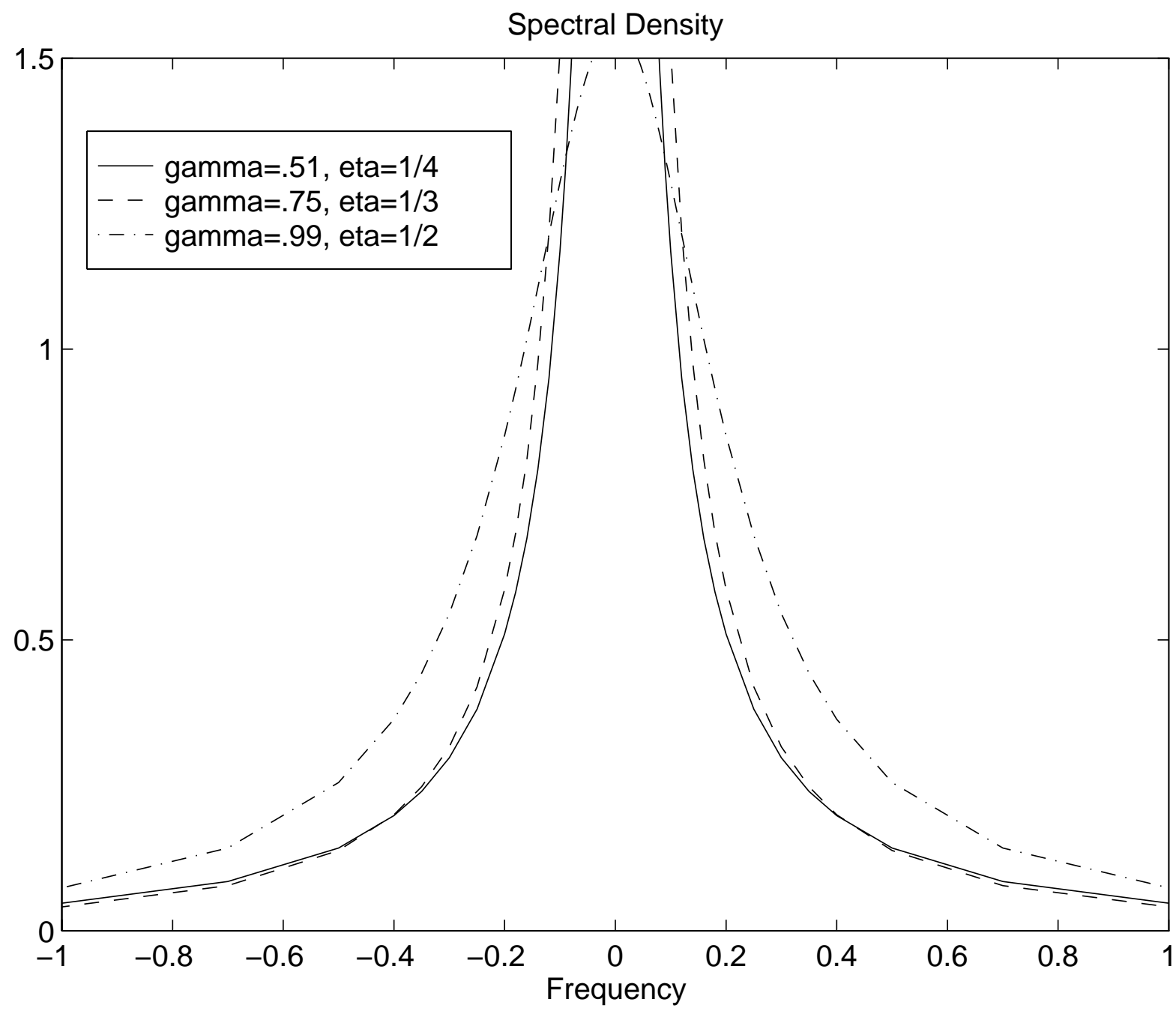

Figure 1: Spectral density functions for different pairs $(\gamma, \eta)$. Spectral densities are rescaled to integrate to one. 


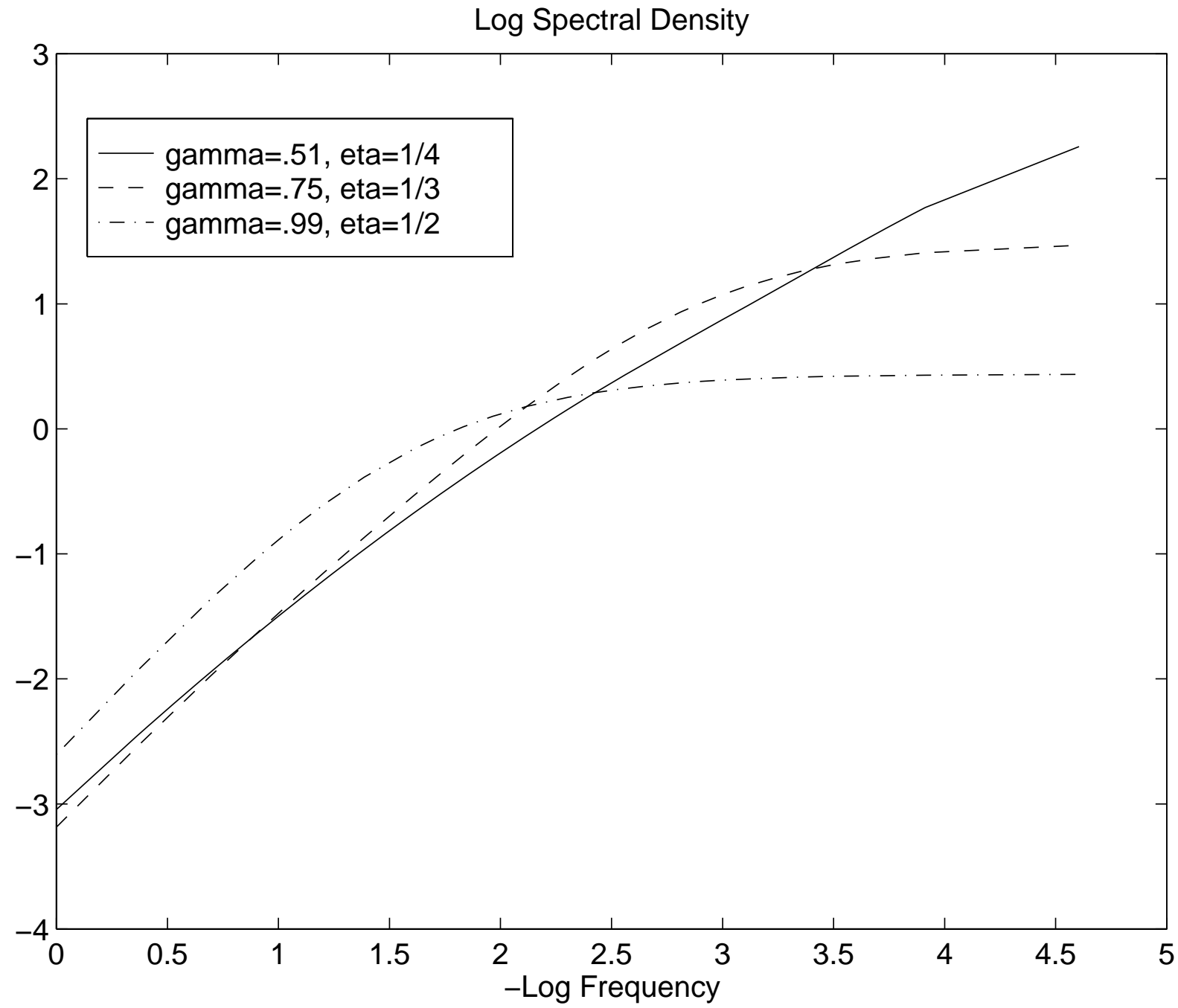

Figure 2: Spectral density functions for different pairs $(\gamma, \eta)$ plotted on a $\log -\log$ scale. Spectral densities are rescaled to integrate to one. 


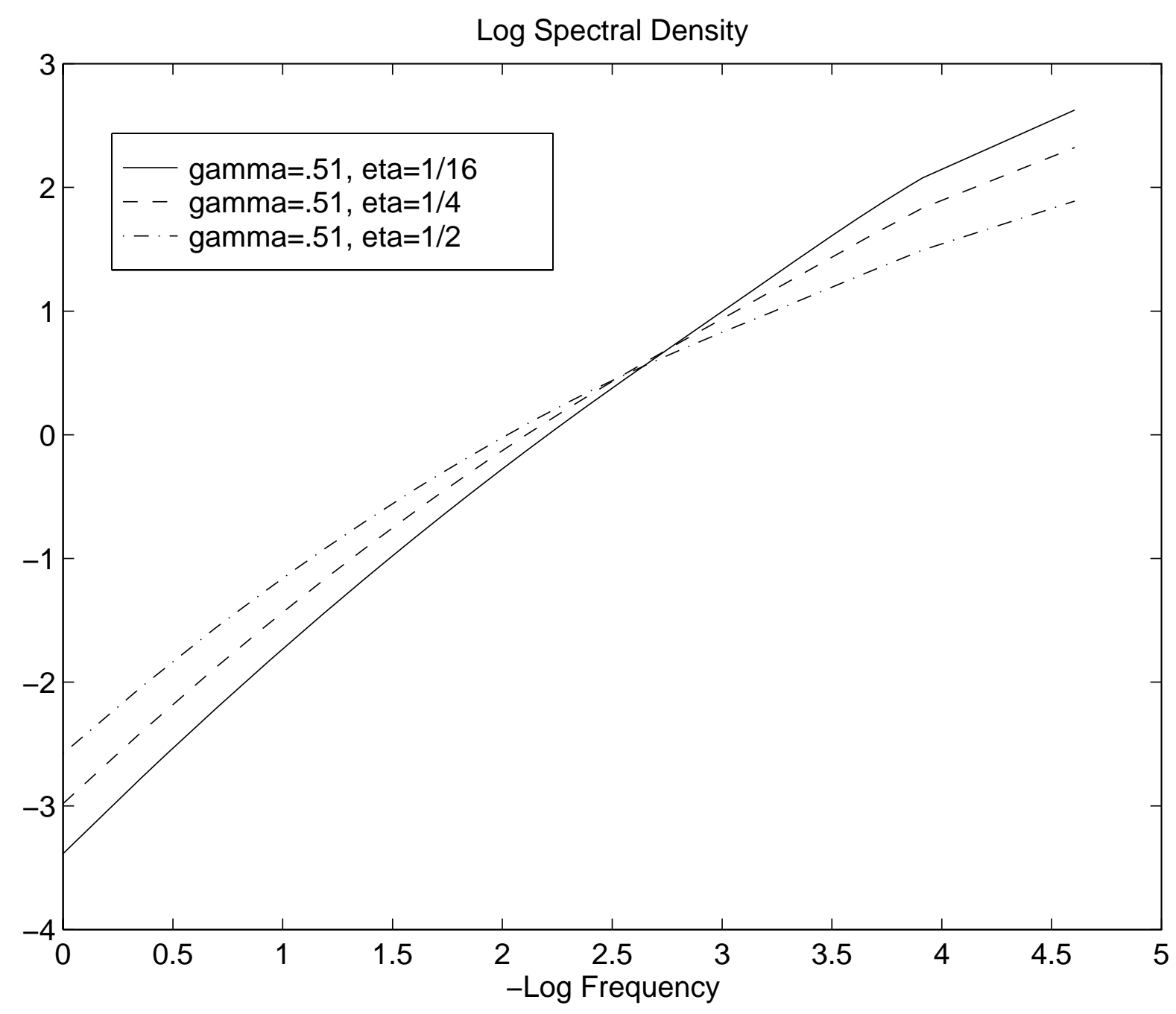

Figure 3: Spectral density functions for different values of $\eta$ plotted on a $\log -\log$ scale. Spectral density functions are rescaled to integrate to one. 
alter the behavior of these functions on say compact subsets and to modify accordingly the transient dynamics for Markov states on compact sets without changing the divergence of the resulting spectral density functions.

Remark 6.2. Stationary versions of fractional Brownian motion are known to have infinite quadratic variations with probability one. As emphasized by Maheswaran and Sims (1993), this feature may make such processes fail to be local martingales and as a consequence unappealing as models of arbitrage-free asset prices. ${ }^{12}$ Maheswaran and Sims (1993) go on to argue that a nice feature of fractional Brownian motions which is often emphasized is its long range dependence and not its "finite-time unit" properties. They then study continuous-time Gaussian moving-average models that break the link between short run responses to shocks and long run dependence. ${ }^{13}$ (Also, see Robinson (1995) for a semiparametric estimation method of models that break this link in a discrete-time setting.) In a similar vein, our nonlinear diffusion examples show how to maintain the local Gaussian structure while inducing nonlinearities and long-run dependence. In particular, the local martingale property is preserved by construction.

\section{Endogenous Sampling}

We conclude the paper by considering discrete-time processes obtained by sampling a diffusion in a manner that is state dependent. Following Duffie and Glynn (2004) we construct an endogenous sampling scheme built from a Poisson process with a state dependent intensity. Let $\left\{x_{t}\right\}$ denote a Markov process with generator $\mathcal{A}$, and let $\left\{N_{t}\right\}$ denote a Poisson process with state-dependent intensity $\lambda$. The event times $\tau_{j}^{*}=\inf \left\{t: N_{t}=j\right\}$ are the times at which $x_{t}$ is observed. We denote the discrete-time process as $\left\{y_{j}^{*}: j=0,1,2, \ldots\right\}$, where $y_{j}^{*}=x_{\tau_{j}^{*}}$, with $\tau_{0}^{*}=0$.

While the intensity $\lambda$ can depend on the Markov state, there is an equivalent way to depict the process $\left\{y_{j}^{*}\right\}$ with an intensity that is state independent. This construction first alters the time clock of the diffusion $\left\{x_{t}\right\}$ in a manner analyzed by Ethier and Kurtz (1986), and then uses a Poisson sampling process with a unit intensity applied to the diffusion with a distorted time clock.

\subsection{Altering the Time Clock}

The continuous-time Markov process $\left\{\widehat{y}_{t}: t \geq 0\right\}$ with a distorted time clock may be constructed as follows:

$$
\widehat{y}_{t}=x_{\tau_{t}}
$$

\footnotetext{
${ }^{12}$ As follows from Harrison and Kreps (1979), Harrison, Pitbladdo, and Schaefer (1984) and Maheswaran and Sims (1993), local martingale or semimartingale implications for security markets are tied directly to the classes of admissible trading rules. Trading rule restrictions are required at the outset to admit even geometric Brownian motions as admissible processes. The more severe the trading rule restrictions, the larger is the class of admissible price processes.

${ }^{13}$ Similar properties have also been investigated by Comte and Renault (1996) for a multivariate continuous-time moving-average models driven by fractional Brownian Motion.
} 
with the increasing process:

$$
\tau_{t}=\int_{0}^{t} \frac{1}{\lambda\left(x_{u}\right)} d u
$$

Assumption 7.1. $\lambda$ is continuous, strictly positive on $(\ell, r)$.

Ethier and Kurtz (1986) (pages 308-309) show that we may construct the Markov process $\left\{\widehat{y}_{t}\right\}$ by using the generator:

$$
\widehat{\mathcal{A}}=\frac{1}{\lambda} \mathcal{A}
$$

motivated heuristically by the chain rule. Since $\lambda$ can be state dependent, the domain of $\widehat{\mathcal{A}}$ may differ from that of $\mathcal{A}$, but the intersection of the domains will typically contain a dense set of functions. While the Ethier and Kurtz (1986) construction is applicable to a general class of Markov processes, we are interested in the case in which the original process is a scalar diffusion with continuous drift and diffusion coefficients, $\mathcal{A}$ and hence $\widehat{\mathcal{A}}$ is a secondorder differential operator that is at least well defined on the space $C^{2}$. The time-deformed process $\left\{\widehat{y}_{t}: t \geq 0\right\}$ is still a diffusion, where the drift and the diffusion coefficients are obtained by multiplying the original drift and diffusion coefficients of $\left\{x_{t}\right\}$ by the reciprocal of the intensity $\lambda$. The scale function of the new process remains unchanged.

The stationary distribution $\widehat{Q}$ of the process $\left\{\widehat{y}_{t}\right\}$ may be constructed as the RadonNikodym derivative proportional to:

$$
d \widehat{Q}=\frac{\frac{\lambda}{s \sigma^{2}}}{\int_{\ell}^{r} \frac{\lambda}{s \sigma^{2}}} .
$$

Given the state dependent intensity $\lambda, \widehat{Q}$ is different from the stationary distribution $Q$ of $\left\{x_{t}\right\}$. In fact one may not even exist while the other one is a positive finite measure. The next assumption ensures the existence of $\widehat{Q}$ :

Assumption 7.2. $\int_{\ell}^{r} \frac{\lambda(x)}{s(x) \sigma^{2}(x)} d x<\infty$.

Now all the results in previous sections apply to the time-altered diffusion $\left\{\widehat{y}_{t}: t \geq 0\right\}$. In particular, the pull measure for the altered process is given by:

$$
\frac{1}{\lambda^{1 / 2}}\left[\frac{\mu}{\sigma}-\frac{\sigma^{\prime}}{2}+\frac{\sigma \lambda^{\prime}}{4 \lambda}\right]
$$

when $\lambda$ and $\sigma$ are differentiable.

\subsection{Poisson Sampling}

We can now form the discrete-time process $\left\{y_{j}^{*}: j=0,1,2, \ldots\right\}$ by taking a Poisson sample of $\left\{\widehat{y}_{t}: t \geq 0\right\}$ with a unit intensity. The resulting discrete-time process is still stationary with distribution $\widehat{Q}$, and is an aperiodic Markov chain with one-period transition operator:

$$
\widehat{\mathcal{T}} \phi(y)=(\mathcal{I}-\widehat{\mathcal{A}})^{-1} \phi(y) .
$$


The discrete-time transition operator is a special case of what is referred to as a resolvent operator for the generator $\widehat{\mathcal{A}}$.

The next result states that $\left\{y_{j}^{*}: j=0,1,2, \ldots\right\}$ preserves all the temporal dependence properties of $\left\{\widehat{y}_{t}: t \geq 0\right\}$.

Theorem 7.3. Suppose that Assumptions 3.1, 3.2 and 7.1 are satisfied. If

$$
\begin{aligned}
& \lim \inf _{x \nearrow^{r}} \frac{s \sigma}{|S| \lambda^{1 / 2}}>0 \\
& \lim \sup _{x \searrow \ell} \frac{s \sigma}{|S| \lambda^{1 / 2}}<0,
\end{aligned}
$$

then: Assumption 7.2 holds and $\left\{y_{j}^{*}: j=0,1,2, \ldots\right\}$ is stationary, $\rho$-mixing and $\beta$-mixing with exponential decay rates.

This theorem is a special case of Theorem A.1, which is stated and proved in the appendix. The latter theorem includes a characterization of the $\beta$ - mixing coefficients when the resulting process is strongly dependent.

Remark 7.4. When $\sigma$ and $\lambda$ are smooth, the sufficient conditions for inequalities (19) and (20) are:

$$
\begin{aligned}
& \limsup _{x \operatorname{sur}^{\prime}} \lambda^{-1 / 2}\left[\frac{\mu}{\sigma}-\frac{\sigma^{\prime}}{2}+\frac{\sigma \lambda^{\prime}}{4 \lambda}\right]<0, \\
& \lim \inf _{x \searrow \ell} \lambda^{-1 / 2}\left[\frac{\mu}{\sigma}-\frac{\sigma^{\prime}}{2}+\frac{\sigma \lambda^{\prime}}{4 \lambda}\right]>0 .
\end{aligned}
$$

Consider now the case in which the subordinated process $\left\{\widehat{y}_{t}\right\}$ is stationary (Assumption 7.2 is satisfied), but its pull measure:

$$
\lambda^{-1 / 2}\left[\frac{\mu}{\sigma}-\frac{\sigma^{\prime}}{2}+\frac{\sigma \lambda^{\prime}}{4 \lambda}\right]
$$

is zero at one of the two boundaries. By the arguments in Hansen, Scheinkman, and Touzi (1998), there exists a sequence of functions $\left\{\phi_{j}\right\}$ with norm one such that:

$$
\lim _{j \rightarrow \infty} \int \phi_{j}\left(\widehat{\mathcal{A}} \phi_{j}\right) d \widehat{Q}=0
$$

It follows that there exists a sequence of functions $\left\{\psi_{j}\right\}$ with mean zero and unit norm such that:

$$
\lim _{j \rightarrow \infty} \int \psi_{j}\left(\widehat{\mathcal{T}} \psi_{j}\right) d \widehat{Q}=1
$$

which implies that all of the discrete-time $\rho$ - mixing coefficients are unity. Hence the dependence properties of the Poisson sampled discrete-time process $\left\{y_{j}^{*}\right\}$ mirror that of the deformed continuous-time process $\left\{\widehat{y}_{t}\right\}$.

Our next examples illustrate how subordination can alter the unconditional distribution as well as the temporal dependence of a scalar diffusion: 
Example 7.5. Let $\left\{x_{t}\right\}$ be a stationary diffusion process on $(-\infty,+\infty)$ with $\mu(x)=-\gamma x$ for some $\gamma>-\frac{1}{2}$ and $\sigma^{2}(x)=1+x^{2}$. The stationary density $q(x)$ is proportional to $\left(1+x^{2}\right)^{-\gamma-1}$ (Example "E" in Wong (1964)). Clearly,

$$
\lim _{x \searrow-\infty} \frac{\mu}{\sigma}-\frac{\sigma^{\prime}}{2}=\gamma+\frac{1}{2}>0, \lim _{x \nearrow+\infty} \frac{\mu}{\sigma}-\frac{\sigma^{\prime}}{2}=-\gamma-\frac{1}{2}<0 .
$$

Thus the $\rho$-mixing and $\beta$-mixing coefficients decay exponentially. Let $\left\{\widehat{y}_{t}\right\}$ be the timedeformed diffusion with $\lambda(x)=1+x^{2}$. Then for $\gamma \in\left(-\frac{1}{2}, \frac{1}{2}\right]$, this diffusion does not have a stationary distribution. While for $\gamma>\frac{1}{2}$ this diffusion has a stationary density proportional to $\left(1+x^{2}\right)^{-\gamma}$, and has a pull measure equal to zero at both boundaries. Thus the $\rho-$ mixing coefficients are unity and the $\beta$-mixing coefficients decay slowly. When we take a Poisson sample of the process $\left\{\widehat{y}_{t}\right\}$ with a unit intensity, the discrete-time process $\left\{y_{j}^{*}\right\}$ remains non-stationary for $\gamma \in\left(-\frac{1}{2}, \frac{1}{2}\right]$, and stationary but strongly dependent for $\gamma>\frac{1}{2}$.

Example 7.6. Suppose that $\left\{x_{t}\right\}$ has the same drift and diffusion coefficient as $\left\{\widehat{y}_{t}\right\}$ had in the previous example: $\mu=\frac{-\gamma x}{1+x^{2}}$ and $\sigma^{2}=1$. As we just argued, this process is stationary and strongly dependent for $\gamma>\frac{1}{2}$, and fails to be stationary when $\gamma \in\left(-\frac{1}{2}, \frac{1}{2}\right]$. Let $\lambda(x)=\frac{1}{1+x^{2}}$, then the resulting time-deformed $\left\{\widehat{y}_{t}\right\}$ process coincides with the $\left\{x_{t}\right\}$ process of the previous example. Hence $\left\{\widehat{y}_{t}\right\}$ and the associated Poisson-sampled discrete-time process $\left\{y_{j}^{*}\right\}$ with a unit intensity are stationary, $\beta$ - mixing and $\rho-$ mixing with exponential decay.

\section{Concluding Remarks}

In this paper we studied the temporal dependence of nonlinear scalar diffusion models. As we have seen, scalar diffusion models provide a convenient and pedagogically valuable platform for understanding how nonlinearities in time series models get transmitted into temporal dependence. Scalar diffusions are of course special. Nevertheless, they are often used as building blocks in more realistic empirical models of security returns. In section 7.1 and in Carrasco, Hansen, and Chen (1999) we study temporal dependence in models of subordinated diffusions. In these models the time clock is distorted in a random and temporally dependent way. Since the work of Clark (1973) and Nelson (1990) it has been known that subordination is a convenient way to model returns with unconditional distributions of fat tails and volatility that is clustered over time. Strongly dependent diffusions may provide a useful tool for studying the time clock distortion or for stochastic volatility models of security market returns. As we noted in the introduction, it is often argued that empirically plausible models of the stochastic volatility factor should be highly persistent.

Strongly dependent diffusions, like models of fractional integration, serve to blur the distinction between stationary and nonstationary processes. As we have seen, the strong dependence of diffusions is conveniently manifested in the pull of the diffusion at extreme values of the Markov state. As a practical matter this pull behavior will be hard to measure accurately without using parametric restrictions on, at the very least, the tail behavior of the drift and diffusion coefficients. This practical problem, however, is no different than what occurs in attempts to detect the degree of long range dependence in times series. It is known that once we allow for flexible transient dynamics, the degree of long range dependence is hard to measure. 


\section{A Appendix}

Proof. (Theorem 3.6): It suffices to prove this result in the natural scale. We establish uniform ergodicity by finding a nonnegative, $C^{2}$ function $V$ that satisfies:

$$
\frac{1}{2} \theta^{2} V^{\prime \prime} \leq-c(V+1)+d \mathbf{1}_{K}
$$

for some positive numbers $c$ and $d$ and some compact set $K$. We construct the function $V$ by first solving the eigenvalue problem:

$$
\frac{1}{2} \theta^{2} \phi^{\prime \prime}=-c \phi
$$

for some $c>0$ and some $\phi \in C^{2}$, and then constructing $V$ from $\phi$. If $\phi$ solves this differential equation than so does $a \phi$ for any real number $a$. Since the diffusion is $\rho-$ mixing, we may choose a sufficiently small $c>0$ such that $\phi$ has only a finite number of zeroes. Let $K$ be a closed interval containing all of the zeroes as interior points. Notice that $\phi$ is concave when it is positive and convex when it is negative. Thus $\phi$ is bounded away from zero in both tails. We let $V+1$ be equal to $a \phi$ to the left of $K$ and $b \phi$ to the right of $K$ where the scale factors are chosen so that $V+1$ exceeds one outside $K$. We extend $V$ to the interior so that it remains nonnegative and is $C^{2}$, and select $d$ to guarantee inequality (21) on $K$.

Proof. (Theorem 3.7): Let $\phi$ be a non-trivial solution of the eigenvalue problem:

$$
\frac{1}{2} \theta^{2} \phi^{\prime \prime}=-c \phi
$$

It suffices to show that $\phi$ has a finite number of zeroes. In the study of second-order differential equations, it is common to use the Prufer substitution (Birkhoff and Rota (1989), page 312) to count the number of zeroes of a solution to a second-order differential equation. We recall the Prufer substitution:

$$
\phi^{\prime}(z)=r(z) \cos \alpha(z), \phi(z)=r(z) \sin \alpha(z)
$$

where

$$
(r(z))^{2}=(\phi(z))^{2}+\left(\phi^{\prime}(z)\right)^{2} ; \alpha(z)=\arctan \left(\frac{\phi(z)}{\phi^{\prime}(z)}\right) .
$$

Obviously $r(z)=0$ for a given $z$ if and only if $\phi^{\prime}(z)=0=\phi(z)$, which leads to a trivial solution $\phi(\cdot) \equiv 0$ for $(22)$. Hence we can assume $r(z)>0$ for all $z$. Then the secondorder differential equation (22) is equivalent to the following system of first-order differential equations for $(r, \alpha)$ :

$$
\begin{aligned}
(\alpha(z))^{\prime} & =\frac{2 c}{\theta^{2}(z)} \sin ^{2} \alpha(z)+\cos ^{2} \alpha(z) \\
(r(z))^{\prime} & =\frac{1}{2}\left[1-\frac{2 c}{\theta^{2}(z)}\right] r(z) \sin 2 \alpha(z)
\end{aligned}
$$


Notice that although $\arctan \left(\frac{\phi(z)}{\phi^{\prime}(z)}\right)$ is not defined whenever $\phi^{\prime}(z)=0$, the Prufer system of first-order differential equations (24)-(25) are well-defined and have unique solution given any initial values say $\left(r\left(z_{0}\right), \alpha\left(z_{0}\right)\right)=\left(r_{0}, \alpha_{0}\right)$, which in turn defines a unique solution $\phi(z)$ for the second-order equation (22) via (23). Moreover, every non-trivial solution $\phi(\cdot)$ to $(22)$ takes value zero at a point $z(\phi(z)=0)$ if and only if the solution $\alpha(\cdot)$ to $(24)$ takes value $n \pi$ for some integer $n$ at that point $z$, (i.e. $\sin \alpha(z)=0$ ).

The equation (24) has a unique solution $\alpha(z)$ for any initial value say $\alpha\left(z_{0}\right)=a$, and the solution is an increasing (continuously differentiable) function. In particular, $\alpha(z)$ is bounded (above and below) over the compact set $K$. Thus $\phi$ has only a finite number of zeroes over the compact set $K$.

We now show that $\phi$ has at most finite many zeros outside the set $K$, (or equivalently, $\alpha(z)$ is bounded above and below outside the set $K)$. Suppose $\phi$ has a zero to the right of $K$. (Otherwise the conclusion follows immediately.) Thus there exists a $z^{*}$ to the right of $K$ such that

$$
\alpha\left(z^{*}\right)=n^{*} \pi \text { for some integer } n^{*} .
$$

Applying the Prufer substitution to inequality (3), and denote the corresponding new dependent variables as $\left(r_{V+1}, \alpha_{V+1}\right)$ (in particular $\left.\alpha_{V+1}(z)=\arctan \left(\frac{V+1}{V^{\prime}}\right)\right)$. It may be shown that

$$
\left(\alpha_{V+1}(z)\right)^{\prime} \geq \frac{2 c}{\theta^{2}(z)} \sin ^{2} \alpha_{V+1}(z)+\cos ^{2} \alpha_{V+1}(z) .
$$

Since $V+1$ never crosses the zero axis, the function $\alpha_{V+1}(z)$ can be initialized to be in the interval $\left(n^{*} \pi,\left(n^{*}+1\right) \pi\right)$ for $z \geq z^{*}$. In particular,

$$
\alpha_{V+1}\left(z^{*}\right)>n^{*} \pi=\alpha\left(z^{*}\right) .
$$

From the Comparison Theorem (e.g. see Birkhoff and Rota (1989), pages 29-31),

$$
\alpha_{V+1}(z) \geq \alpha(z) \quad \text { for all } z \geq z^{*}
$$

implying that

$$
\alpha(z)<\left(n^{*}+1\right) \pi \quad \text { for all } z \geq z^{*}
$$

Hence $\phi$ has no zero values for all $z>z^{*}$. An analogous argument that studies the behavior of $\alpha(z), \phi(z)$ to the left of the set $K$.

Proof. (Theorem 5.1): We follow Lindvall (1983) and use a coupling argument. Consider two independent diffusions. One $\left\{z_{t}^{1}: t \geq 0\right\}$ is initialized at $z$ and the other is initialized according to the stationary distribution. We are interested in the stopping time $\tau \equiv \inf \{t \geq$ $\left.0: z_{t}^{1}=z_{t}^{2}\right\}$. The probability distribution for $z_{t}^{1}$ and $z_{t}^{2}$ coincide from $\tau$ on. Define the conditional $\beta$-mixing coefficient:

$$
\beta_{t}(z) \equiv \sup _{0 \leq \phi \leq 1}\left|E\left[\phi\left(z_{t}^{1}\right) \mid z_{0}^{1}=z\right]-E \phi\left(z_{t}^{2}\right)\right|
$$

Then from Lindvall (1983) (Section 2),

$$
\beta_{t}(z) \leq \operatorname{Pr}\left\{\tau>t \mid z_{0}^{1}=z\right\} .
$$


As a consequence,

$$
\beta_{t} \leq E\left[\beta_{t}\left(z_{0}^{1}\right)\right] \leq \operatorname{Pr}\{\tau>t\} .
$$

To bound the tail probabilities of the hitting time $\tau$, we follow Pitman (1974) and Lindvall (1983) by using a familiar inequality for nonnegative random variables. Suppose that $E(\xi(\tau))<\infty$ for $\xi \geq 0$ non-decreasing on $[0, \infty)$. Then

$$
\lim _{t \uparrow \infty} \xi(t) \operatorname{Pr}\{\tau>t\}=0 \text { hence } \lim _{t \uparrow \infty} \xi(t) \beta_{t}=0 .
$$

If further, $\xi()$ is absolutely continuous with respect to Lebesgue measure and has density $(\xi)^{\prime}$, then a simple integration-by-parts argument implies that

$$
\int_{0}^{\infty}(\xi(t))^{\prime} \operatorname{Pr}\{\tau>t\}<\infty \text { hence } \int_{0}^{\infty}(\xi(t))^{\prime} \beta_{t}<\infty .
$$

Inequalities (8) now follow from setting $\xi(t)=t^{\delta}$ for some $\delta>0$.

Theorem A.1. Suppose that Assumptions 3.1, 3.2 and 7.1 are satisfied. If for some $\eta \in$ $\left(\frac{1}{2}, 1\right]$,

$$
\begin{aligned}
& \lim \inf _{x \nearrow r} \frac{s \sigma}{|S|^{\eta} \lambda^{1 / 2}}>0 \\
& \lim \sup _{x \searrow \ell} \frac{s \sigma}{|S|^{\eta} \lambda^{1 / 2}}<0,
\end{aligned}
$$

then: (i) Assumption 7.2 holds and $\left\{y_{j}^{*}: j=0,1,2, \ldots\right\}$ is stationary $\beta-$ mixing for $\eta>\frac{1}{2}$. Denote

$$
\eta^{*} \equiv \sup \left\{\eta \in\left(\frac{1}{2}, 1\right]: \text { inequalities (19) and (20) are satisfied }\right\} .
$$

(ii) If $\eta^{*}=1$, then $\left\{y_{j}^{*}: j=0,1,2, \ldots\right\}$ is $\rho$-mixing and $\beta$-mixing with exponential decay rates; (iii) if $\eta^{*} \in\left(\frac{1}{2}, 1\right)$, then $\lim _{j \rightarrow \infty} j^{\delta} \beta_{j}=0$ for any $\delta<\delta^{*}$ where $\delta^{*}=\frac{2 \eta^{*}-1}{2-2 \eta^{*}}$.

Proof. (Theorem A.1): In what follows we let $\widehat{D}$ denote the domain of $\widehat{\mathcal{A}}$ constructed using the stationary distribution $\widehat{Q}$, and $\widehat{L}^{2}$ denote the space of functions with finite second moment (against $\widehat{Q}$ ).

(i) As long as $\eta>\frac{1}{2}$, Assumptions 3.1, 3.2 and 7.1 imply that the time-altered continuous time diffusion $\left\{\widehat{y}_{t}: t \geq 0\right\}$ is stationary, recurrent and aperiodic. Hence $\left\{y_{j}^{*}: j=0,1,2, \ldots\right\}$ is still stationary, recurrent and aperiodic; hence it is $\beta$ - mixing.

(ii) If $\eta=1$ in inequalities (19) and (20), then $\left\{\widehat{y}_{t}: t \geq 0\right\}$ is $\rho$-mixing and $\beta$-mixing with exponential decay by Corollary 4.3.

Next, by the result of Banon (1977) (see also Hansen and Scheinkman (1995)), $\rho$-mixing of $\left\{\widehat{y}_{t}: t \geq 0\right\}$ implies the existence of spectral gap of the negative semidefinite generator $\widehat{\mathcal{A}}$. That is, $\widehat{\mathcal{A}}$ satisfies

$$
\int \phi(\widehat{\mathcal{A}} \phi) d \widehat{Q} \leq-\delta \int \phi^{2} d \widehat{Q}
$$


for all $\phi \in \widehat{Z}$ for some $\delta>0$ where

$$
\widehat{Z}=\left\{\phi \in \widehat{D}: \int \phi d \widehat{Q}=0\right\}
$$

An implication of (26) is that

$$
\int \phi(\mathcal{I}-\widehat{\mathcal{A}}) \phi d \widehat{Q} \geq(1+\delta) \int \phi^{2} d \widehat{Q}
$$

Therefore, by equation (18)

$$
\int \phi \widehat{\mathcal{T}} \phi d \widehat{Q} \leq \frac{1}{1+\delta} \int \phi^{2} d \widehat{Q}
$$

for $\phi \in \widehat{Z}$. In other words, the conditional expectation operator $\widehat{\mathcal{T}}$ of the discrete time process $\left\{y_{j}^{*}: j=0,1,2, \ldots\right\}$ is a strong contraction on $\phi \in \widehat{Z}$. Since $\widehat{D}$ is dense in $\widehat{L}^{2}$, it follows from Rosenblatt (1971) that $\left\{y_{j}^{*}: j=0,1,2, \ldots\right\}$ is $\rho$ - mixing with exponential decay provided that inequality (26) is satisfied, which holds given inequalities (19) and (20) with $\eta=1$.

Finally, by Theorem 3.6, the $\rho-$ mixing of $\left\{\widehat{y}_{t}: t \geq 0\right\}$ also implies that there exists a non-negative Lyapunov function $V \in C^{2}$ with $V \geq 1$, a compact set $K$, positive constants $c$ and $d$ such that:

$$
\widehat{\mathcal{A}} V \leq-c V+d \mathbf{1}_{K}
$$

Thus:

$$
(\mathcal{I}-\widehat{\mathcal{A}}) V \geq(1+c) V-d \mathbf{1}_{K}
$$

Take inverses and obtain:

$$
V \geq(1+c)(\mathcal{I}-\widehat{\mathcal{A}})^{-1} V-d \mathbf{1}_{K}
$$

or

$$
\widehat{\mathcal{T}} V(y) \leq \frac{1}{1+c} V(y)+\frac{d}{1+c} \mathbf{1}_{K}
$$

We may now apply theorem 2.1 of Down, Meyn, and Tweedie (1995) to justify that $\left\{y_{j}^{*}: j=0,1,2, \ldots\right\}$ is $\beta-$ mixing with exponential decay.

(iii) If $\eta \in\left(\frac{1}{2}, 1\right]$ in inequalities (19) and (20), then by Theorem 5.2, $\left\{\widehat{y}_{t}: t \geq 0\right\}$ is $\beta-$ mixing with $\lim _{t \rightarrow \infty} t^{\delta} \beta_{t}=0$. To establish the result for $\left\{y_{j}^{*}: j=0,1,2, \ldots\right\}$, we apply the theorems 2.3 and 4.3 of Tuominen and Tweedie (1994), which is for discrete-time Markov processes.

\section{References}

Andersen, T., T. Bollerslev, F. X. Diebold, and P. Labys (2001). The distribution of realized exchange rate volatility. Journal of American Statistical Association 96, 4255.

Banon, G. (1977). Estimation Non Paramétrique de Densité de Probabilité pour les Processus de Markov. Ph. D. thesis, Université Paul Sabatier, Toulouse, France. 
Bhattacharya, R. N. (1982). On the functional central limit theorem and the law of the iterated logarithm. Zeitschrift für Wahrscheinlichkeitstheorie und verwandte Gebiete 60, $185-201$.

Birkhoff, G. and G.-C. Rota (1989). Ordinary Differential Equations. Wiley \& Sons.

Bollerslev, T. and H. O. Mikkelsen (1996). Modeling and pricing long memory in stock market volatility. Journal of Econometrics 73, 151-184.

Bradley, R. (1986). Basic properties of strong mixing conditions. In E. Eberlein and M. S. Taqqu (Eds.), Dependence in Probability and Statistics.

Carrasco, M., L. P. Hansen, and X. Chen (1999). Time deformation and dependence. University of Chicago.

Chen, X., L. P. Hansen, and J. Scheinkman (2000). Principal components and long run implications of multivariate diffusions. University of Chicago.

Clark, P. K. (1973). A subordinated stochastic process model with finite variance for speculative prices. Econometrica 41, 135-155.

Comte, F. and E. Renault (1996). Long memory continuous time models. Journal of Econometrics 73, 101-149.

Conley, T. G., L. P. Hansen, E. G. J. Luttmer, and J. A. Scheinkman (1997). Short-term interest rates as subordinated diffusions. The Review of Financial Studies 10, 525-577.

Davydov, Y. (1973). Mixing conditions for markov chains. Theory of Probability and Its Applications, 312-328.

Diebold, F. and A. Inoue (2001). Long memory and regime switching. Journal of Econometrics 105, 131-159.

Ding, Z., C. W. J. Granger, and R. F. Engle (1993). A long-memory property of stock returns and a new model. Journal of Empirical Finance 1, 83-106.

Down, D., S. P. Meyn, and R. L. Tweedie (1995). Exponential and uniform ergodicity of markov processes. The Annals of Probability 23, 1671-1691.

Duffie, D. and P. Glynn (2004). Estimation of continuous-time markov processes sampled at random time intervals. Econometrica (6), 1773-1808.

Ethier, S. and T. Kurtz (1986). Markov Processes. New York: Wiley \& Sons.

Genon-Catalot, V., T. Jeantheau, and C. Laredo (2000). Stochastic volatility models as hidden markov models and statistical applications. Bernoulli 6(6), 1051-1079.

Granger, C. W. J. and T. Teräsvirta (1999). A simple nonlinear time series model with misleading linear properties. Economics Letters 62, 161-165.

Hansen, L. P. and J. Scheinkman (1995). Back to the future: Generating moment implications for continuous-time markov processes. Econometrica 63, 767-804.

Hansen, L. P., J. Scheinkman, and N. Touzi (1998). Spectral methods for identifying scalar diffusions. Journal of Econometrics 86, 1-32.

Harrison, J. M. and D. Kreps (1979). Martingales and arbitrage in multiperiod securities markets. Journal of Economic Theory 20, 381-408. 
Harrison, J. M., R. Pitbladdo, and S. M. Schaefer (1984). Continuous price processes in frictionless markets have infinite variation. Journal of Business 57, 353-365.

Has'minskii, R. Z. (1980). Stochastic Stability of Differential Equations. Sijthoff \& Noordhoff.

Hidalgo, J. and P. Robinson (1996). Testing for structural change in a long-memory environment. Journal of Econometrics 70, 159-174.

Ibragimov, I. A. and Y. A. Rozanov (1978). Gaussian Random Processes. New York: Springer-Verlag.

Lindvall, T. (1983). On coupling of diffusion processes. Journal of Applied Probability 20, 82-93.

Maheswaran, S. and C. A. Sims (1993). Empirical implications of arbitrage-free asset markets. In P. C. B. Phillips (Ed.), Models, Methods, and Applications of Econometrics: Essays in Honor of A. R. Bergstrom, pp. 301-316.

Mandelbrot, B. B. and J. W. V. Ness (1968, October). Fractional brownian motions, fractional noises, and applications. SIAM Review 10.

Meyn, S. P. and R. L. Tweedie (1993). Stability of markovian processes iii: Foster-lyapunov criteria for continuous-time processes. Advances in Applied Probability 25, 518-548.

Nelson, D. (1990). Arch models as diffusion approximations. Journal of Econometrics 45, $7-39$.

Pitman, J. W. (1974). Uniform rates of convergence for markov chain transition probabilities. Zeitschrift für Wahrscheinlichkeitstheorie und verwandte Gebiete 29, 193-227.

Robinson, P. M. (1995). Log-periodogram regression for time series with long-range dependence. Annals of Statistics 23, 1048-1072.

Rosenblatt, M. (1971). Markov Processes, Structure and Asymptotic Behavior. SpringerVerlag.

Tuominen, P. and R. L. Tweedie (1994). Subgeometric rates of convergence of f-ergodic markov chains. Advances in Applied Probability 26, 775-798.

Veretennikov, A. Y. (1987). Bounds for the mixing rate in the theory of stochastic equations. Theory of Probability and its Applications 32, 273-281.

Veretennikov, A. Y. (1997). On polynomial bounds for stochastic differential equations. Stochastic Processes and their Applications 70, 115-127.

Volkonskii, Y. A. and Y. Rozanov (1959). Some limit theorems for random functions i. Theory of Probability and its Applications 4, 178-197.

Weidmann, J. (1987). Spectral Theory of Ordinary Differential Operators. New York: Springer-Verlag.

Wong, E. (1964). The construction of a class of stationary markoff processes. In R. Bellman (Ed.), Sixteenth Symposium in Applied Mathematics-Stochastic Processes in Mathematical Physics and Engineering, pp. 264-276. Providence, RI: Americal Mathematical Society. 